\title{
Water bird fauna in the Carpathian Basin from the beginnings through historical times
}

\author{
Jenö (Eugen) KESSLER
}

Received January 16, 2017 - Accepted April 24, 2017

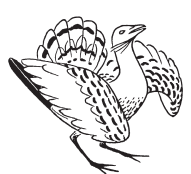

Kessler, J. 2017. Water bird fauna in the Carpathian Basin from the beginnings through historical times. - Ornis Hungarica 25(1): 70-100. DOI: 10.1515/orhu-2017-0006. of avian fauna near waterbodies, since ornithologists, who rarely come across or research the paleontology of birds, do not possess significantly detailed knowledge about the evolution and evidence of the current avian fauna.

Keywords: birds, waterfowls, evolution, aquatic environments, Carpathian Basin

\begin{abstract}
Összefoglalás „A Kárpát-medencei vizek madárvilága a kezdetektől a történelmi időkig” tanulmányban a szerző összefoglalja mindazt, amit a vízi élőhelyek madárvilágának evolúciójáról, fosszilis leleteiről jelenleg tudunk. Azért tartottuk szükségesnek mindezt megírni, mivel a madarak őslénytanával nemigen foglalkozó, és vele ritkán találkozó ornitológusok nem rendelkeznek túl gazdag ismeretekkel a mai madárvilág kialakulásáról és annak bizonyítékairól.
\end{abstract}

Kulcsszavak: madarak, vízi madárvilág, evolúció, vízi környezet, Kárpát-medence

Department of Paleontology, Eötvös Loránd University, 1117 Budapest, Pázmány Péter sétány 1/c, Hungary, e-mail: kessler_jeno@yahoo.com

\section{Introduction}

Aquatic and wet environments have always provided ample living conditions for birds, even when the surface merely consisted of snow and ice fields (like Antarctica), steep cliffs, or bare beaches or sandbanks. This is due to the fact that birds can acquire their food supply from water, while the relatively dry area necessary for reproduction is provided by the environments listed. Some aquatic bird species downright build their nests onto the water. Due to their constant body temperature and usually waterproof feathers, the climate of the environment only influences their abundance through the amount of nutrition they and their offspring require. In case of low nutrient supply, the birds are forced to migrate. It concludes from the above that they mostly consume other animals, however, exclusively herbivores are also included (such as geese).

They usually live in pairs which can last for their entire lifetime, but there are also polygamous species. Nests can be absent altogether (as with the penguins of Antarctica), or can be quite simple (consisting of a few pebbles and sticks), but can even be carefully prepared and expanded for years (as with storks) or formed out of mud (as with flamingos). The number of nestlings can vary from one to a dozen, and can either be nidifugous or incessores. 
Based on their way of lives they can be swimmers, leaping into water from heights, waders, and species living at the shore or in wet environments:

- swimmers can propel themselves either with their wings (penguins, razorbills, petrels) and can usually dive more easily and can spend more time underwater, or with their legs (Hesperornis, divers, grebes, pelicans, cormorants, geese, Anatidae etc.);

- seabirds usually leap into the water from great heights to catch their prey (gannets, petrels, razorbills etc.);

- waders stand and walk shallow waters to collect aquatic animals with their long beaks;

- species living on the shore are usually smaller in size, rarely venture to open waters, and collect their nutrition from the flora or coastal sand;

- species living in wet environments are tied more to the wildlife of where they live, nest and feed, but they don't actually require large surface of water.

From the Jurassic-Cretaceous boundary, the findings of the above-mentioned types emerge fairly early among recent birds. In fact, they make up the majority of fossilized remains of avian fauna of the Cretaceous period (Ornithure - Neornithes). Whether this has taphonomic reasons or they in fact constituted the majority of this early phase of avian fauna could not be proven at that time, but all signs point to this.

Those belonging to taxons outside of the species of orders featured in the part describing taxonomy can also be tied to near-water environments (osprey, kingfisher, dipper etc.), but they are not the subject of this paper.

According to their relation to aquatic environments, we can distinguish between swimmers (dabblers, divers), waders, those living on the shore and those living in wet environments. The fluffy or naked skin of nestlings, and their incessorous or nidifugous nature also indicates their primary or secondary connections with water. Their body covered in down feathers, the early leaving of their nests and their ability to feed on their own all indicate their ancient characteristics still present from the reptilian origins.

Since in case of extinct taxons, we cannot know the exact environments in which they lived, we are also going to discuss groups or species that probably were not that partial to aquatic environments, but their place in taxonomy classifies them as belonging to this category.

The paper, of course, is not all-encompassing, thus, we are only going to mention the most representative taxons. We found it necessary, however, to also include data of recent avian fauna of the Carpathian Basin to present the formation and relations of recent fauna in the Quarter from the past few hundreds of thousands of years.

\section{Taxonomy}

Since the genome of extinct species was lost during fossilization, their inclusion in more recent, DNA-based systems is not possible (Sybley \& Ahlquist 1990, Sybley \& Monroe 1990, Jarvis et al. 2014) neither from a practical, nor from a theoretical point of view. From the practical standpoint, there is no definitive DNA to compare, and from the theoretical standpoint, there is no way to distinguish between analogous features formed by convergence and 
homologous features passed on from their ancestors. Due to these shortcomings, we are going to use the old system of classification (Wetmore 1960), which includes extinct groups (orders, families). In the past decades, a number of revisions synonymized or reclassified rather many previously classified species into different taxons. We took these changes into account by necessity, but did not agree with them in all cases, aiming at a classification as transparent as possible.

Data was extracted primarily from the works of Brodkorb $(1963,1964,1967)$, Olson (1977, 1985), Kurochkin (1995, 2006), Mourer-Chauviré (1995), Mlikovský (1996, 2002), Tyrberg (1998), Feduccia (1999) and Mayr (2009), complemented with other sources (see bibliography) and with data from other works of this paper's author. References are thus kept to those absolutely necessary.

It is worth noting that families that have no known ancestors from prehistoric times are not featured in the listing.

Abbreviations: Q1-Q2 - lower Pleistocene; Q3 (Q3/I-Q3/II) - middle Pleistocene; Q4/I - upper Pleistocene; Q4/II - Holocene; + - Quaternary extinct/fossil species. Cl. - Class; Scl. - Subclass; Ord. - Order; Subord. - Suborder; Fam. - Family; Subfam. - Subfamily.

Cl. Aves (Linneaus, 1758)

Scl. Ornithurae (Haeckel, 1866)

Ord. Hesperornithiformes (Sharpe, 1899)

These birds were present from the beginning of the Cretaceous and typical to the era. Their vestigial wings suggest that even their earliest known representatives (145 million years ago) were already highly specialized, thus, they had to be formed much earlier than that. However, they match with recent birds in almost all known skeletal characteristics (Ornithurae - Neornithes), differing from Sauriurae birds, which are also contemporaries.

\section{Fam. Hesperornithidae (Sharpe, 1899)}

Aquatic birds with vestigial wings, toothed beaks, with a similar constitution to that of Gavia (loons), but of a much bigger size. Dyke and his colleagues reported Hesperonis remains not identified to the species level (Hesperornithidae indet.) in 2010 from the lower Cretaceous of Cornet in Transylvania (Romania) (Dyke et al. 2010).

Hesperornithidae are typical aquatic birds - propelling themselves with their legs - of the Cretaceous period that already have vestigial wings (only the humerus remained, albeit in a deformed form). They lived from the lowermost to the uppermost Cretaceous in several parts of the globe. Their largest representatives were on par, and even bigger than nowadays pelicans. Since their legs were located in the rear, their movement on solid ground was most likely poor. They probably ate fish in waters near shores similarly to recent foot-propeled birds, and this must have resulted in the degradation of their vestigial wings. At the end of the Cretaceous, they went extinct along with Archaeornithae, Enantiornithae and dinosaurs.

\section{Ord. Sphenisciformes (Sharpe, 1891)}

They constitute the most special group within the present avian fauna both in terms of morphology and lifestyle. Most of their characteristics have rather ancient features, which suggest the early development of the order, even though their earliest remains come from the beginning of the Tertiary. 


\section{Fam. Spheniscidae (Bonaparte, 1831)}

The origin and biological relatives are matters of debate. Genetically - debatably - they are tied to seabirds. Nowadays, they are only present on the Southern Hemisphere on shores along cold streams. They feed on fish and pelagic crabs (krill). Due to their typical morphological characteristics (the nature of feathers, lack of cervical apteria, the lack of pneumaticity in bones, the existence of the paleopulmo), they differ from every other group of birds. Their earliest - although contested - representative is Palaeocursornis biharicus Kessler et Jurcsák, 1986 from the Lower Cretaceous of Transylvania (Cornet, Romania) [new combination: Protospheniscus biharicus (Kessler et Jurcsák, 1986), (Kessler, J. 2013)].

\section{Ord. Gaviiformes Wetmore et Miller, 1926}

Loons are typically aquatic birds, with well-developed swimming legs in the rear.

\section{Fam. Gaviidae Allen, 1897}

Fish-eating birds tied to water, they paddle with their legs after diving underwater. They only live on seashores of northern parts of the Northern Hemisphere, only visiting non-freezing freshwater bodies inland in winter. In the Carpathian basin, we knew a questionable Gavia sp. remain from the late Oligocene of Máriahalom, Hungary (Kessler \& Rabi manuscript), as well as an extinct species from the middle Miocene: Gavia schulzii Mlikovský, 1998 from Austria (Mlikovský 1998). Recent species from the Carpathian Basin and Europe are known since the Quaternary:

- Gavia stellata Pontoppidan, 1753

Known from the Carpathian Basin: Q4/II: Kazánszoros-Töröklik (Cuina Turcului Cave-Cazanele), Romania (Kessler 1974a).

- Gavia arctica (Linnaeus, 1758)

Known from the Carpathian Basin: Q4/II: Kőtelek-Huszársarok, Hungary (Jánossy 1979b).

\section{Ord. Podicipediformes (Fürbringer, 1888)}

Grebes are similar in appearance, swimming and lifestyle to loons.

\section{Fam. Podicipedidae Bonaparte, 1831}

They are present on every continent except Antarctica, mostly near freshwater, but certain species also live on the seashore. Their fossil species are known - except the debated $E u$ rolimnornis corneti Kessler and Jurcsák, 1986 (Lower Cretaceous, Transylvania - Cornet, Romania) (Kessler \& Jurcsák 1986) - from the late Oligocene to the Pliocene.

Extinct genera and species of the family are represented by Podiceps csarnotanus Kessler, 2009 from the middle Pliocene of Csarnóta 2, Hungary (Kessler, E. 2009a).

Recent Podiceps species are known since the Quaternary.

- Podiceps nigricollis Brehm, 1831

Known from the Carpathian Basin: Q2: Nagyharsány-hegy 1-4, Hungary (Jánossy 1979a, 1979b).

- Podiceps griseigena (Boddaert, 1783)

Known from the Carpathian Basin: Q4/II: Ludas-Budzsák, Hungary; Remetelórév-Bólyikő Cave (Piatra Boiului-Lorău); Kazánszoros-Töröklik (Cuina Turcului Cave-Cazanele); Vargyasi-szoros Caves (Vârghiṣ Defile) (all in Romania) (Bökönyi 1974, Gál 2004, Kessler 1974, 1982, 1985). 
- Podiceps auritus (Linnaeus, 1758)

Known from the Carpathian Basin: Q4/I: Endrőd 119; Pilisszántó (all in Hungary) (Jánossy 1979a, 1979c, 1985, Gál 2004, 2007b).

- Podiceps ruficollis (Pallas, 1764)

Known from the Carpathian Basin: Q4/II: Maroslele-Pana, Hungary (Jánossy 1979c).

- Podiceps cristatus (Linnaeus, 1758)

Known from the Carpathian Basin: Q4/II: Ecsegfalva 23, Endröd 3/6, Gyula-Castle, Maroslele-Pana, Ludas-Budzsák, Nagykörü-Tsz, Tiszalúc-Sarkad, Tiszavasvári-Deákhalmi-dülö (all in Hungary) (Bökönyi 1974, Jánossy 1979c, 1985, Gál 2004, 2007b, Pike-Tay et al. 2004).

\section{Ord. Procellariiformes Fürbringer, 1888}

Seabirds typically spending their whole - usually long - lives above the open sea and are only on ground when nesting or raising the nestlings.

Fam. Diomedeoididae (Fischer, 1985)

Apart from the four recent families of seabirds, we also know of an extinct family, described also from the Upper Oligocene of Hungary, based on a femur wing imprint that surfaced from the late clay mines of Szépvölgy, Buda, from deep sea sediments and named Diomedeoides harmathi Kessler, 2009 (Kessler, E. 2009).

\section{Ord. Pelicaniformes Sharpe, 1891}

Pelecaniformes are typically aquatic birds, they even gather their food from the water. Their incessorous, bare nestlings, however, show that this attachment to water may be a secondary characteristic in their case.

Fam. Elopterygidae Andrews, 1913

Remains with debatable affiliation from Upper Cretaceous of the Hátszegi Basin (Transylvania, Romania) was discovered by Ferenc Nopcsa, who then sold it to the British Museum, where C. A. Andrews classified and described it as Elopteryx nopcsai Andrews, 1913 (Andrews 1913). Two, once again British, paleornithologists in the seventies of the last century thought to have discovered two large owls in them based on the bones (Heptasteornis andrewsi and Bradycneme draculae Harrison et Walker, 1975) (Harrison \& Walker 1975). The remains of a distal humerus discovered in 2005 in the presence of the author also in Szentpéterfalva (Sânpetru, Romania, Haţeg Basin) decided that it actually belongs to the ancient Pelicans described by Andrews, which thus should be a valid species, despite it being viewed as Troodontidae Alvarezsauridae, or therapod dinosaur (Kessler et al. 2005).

\section{Fam. Pelicanidae Vigors, 1825}

The largest species of the order belong to the family of Pelicans. Except the polar regions, they can be found on every continent, on larger bodies of freshwater, as well as the sea. They eat fish. They nest in reed beds and on the coastal sand of flat islands, forming large groups. Recent species from the Carpathian Basin are known since the Quaternary. Extinct species are not known hence.

- Pelecanus onocrotalus Linnaeus, 1768

Known from the Carpathian Basin: Q4/II: Ács-Vaspuszta, Bajcsa-Castle, Békés-Városerdő, Maroslele-Pana, Zalaszentistván (all in Hungary); Peterd-Tordai-hasadék (Cheile Turzii-Defile, Petrești), Romania; Padina, Serbia (Jánossy 1979c, Classon 1980, Bartosiewicz 1991, Kessler \& Gál 1998, Gál 2002, 2004). 
- Pelecanus sp.

Known from the Carpathian Basin: Q4/II: Szegvár-Tüzköves, Hungary; Kazán-szoros-Töröklik (Cuina Turcului Cave-Cazanele), Parác (Parța) (all in Romania) (Kessler 1974, 2009a, Jánossy 1985, Kessler \& Gál 1997, Gál 2004, 2007b).

\section{Fam. Phalacrocoracidae (Bonaparte, 1854)}

Cormorants can be found on most continents except Antarctica, Middle and Northern Asia, on larger bodies of freshwater, as well as seashores. Cormorants have dark - but not waterproof - feathers, and characteristic beaks. They mostly eat fish, and even though they paddle with their feet, they are also exceptional divers, diving into the water from even great heights. They live in large groups and nest on branches of trees, cliffs or the coastal sand. Their earliest extinct finds are also from the late Cretaceous and Eocene, but their classification is uncertain and debatable. Recent species from the Carpathian Basin are known since the Quaternary. Extinct species are not known hence.

- Phalacrocorax carbo Shaw et Nodder, 1801

Known from the Carpathian Basin: Q4/II: Endrőd 3/6, Röszke-Lúdvár, TiszalucDankadomb (all in Hungary); Parác (Parța), Püspökfürdő Lake (Băile Episcopești) (all in Romania) (Kessler 1974, Jánossy 1979c, 1985, Kessler \& Gál 1997, Gál 2004, 2007b).

\section{Fam. Sulidae (Reichenbach, 1849)}

Boobies and gannets are large to middle-sized fish-eating seabirds, catching their prey by diving from heights. They form large groups on the cliffs and coastal sand of sea shores, and their guano may accumulate into thick layers. Their earliest finds are known from the middle Eocene (Eostega lebedinskyi Lambrecht, 1927 from Transylvania, Romania) and Microsula pygmaea (Milne-Edwards, 1874) from middle Miocene of Austria (Göhlich 2003). The fossil species from Transylvania was described by Lambrecht (Lambrecht 1929 ) based on a jawbone, and according to the revision, this was indeed correct (Mlikovský 2007).

\section{Fam. Anhingidae Reichenbach, 1849}

Snakebirds today are tropical, freshwater birds, harpooning fish with their long, sharp beaks. They nest on trees along the coast. Fossil remains are rather rare in Europe, they are only known form the Carpathian Basin (named Anhinga pannonica Lambrecht, 1916 and Anhinga sp.) from the late Miocene of Tataros in Transylvania (Tătăruṣ), Romania (Lambrecht 1916b), Götzendorf, Austria (Mlikovský 1992), and from the middle Miocene of Mátraszőlős, Hungary (Gál et al. 1998-1999).

\section{Fam. Phaethontidae (Bonaparte, 1853)}

Tropicbirds, as their name suggests, live at tropical seas, with a medium-sized and typical tail feathers. Their diet consists of fish. They prepare their nests inside cavities of cliffs on rocky shores. Two extinct species are known in the Carpathian Basin: Heliadornis paratethydicus Mlikovský, 1997 from late Miocene of Austria (Mlikovský 1997), while H. minor Kessler, 2009 is known from the late Pliocene of Ajnácskő (Hajnačka), Slovakia (Kessler, J. 2009a).

\section{Ord. Ardeiformes (Wagler, 1930)}

Waders (herons, storks, ibises, flamingos) form a group with a rather distinctive form and lifestyle. They have long beaks, necks and legs. They live in wet environments all across 
the globe except the polar regions, feeding on invertebrates living in water or on dry land, fish, and other smaller vertebrates. They live in groups and prepare their nests on trees and reed beds, except for flamingos, who build their tower-like nests out of mud on lake shores with shallow waters.

\section{Subord. Phoenicopteri Fürbringer, 1888}

Fam. Palaelodidae (Stejneger, 1885)

Representatives of the order still alive today are the flamingos, well-known from the typical shape of beaks and pink feathers. They feed on tiny krill or single-celled algae in highly alkaline still waters. They inhabit tropical and subtropical areas. The extinct species Palaelodus goliath Milne-Edwards, 1863 was reported from the middle Miocene of Hungary (Mátraszőlős) (Gál et al. 2000).

\section{Subord. Plataleae Newton, 1884}

\section{Fam. Threskiornithidae Richmond, 1917}

This family contains ibises and spoonbills. Recent species from the Carpathian Basin are known since the Quaternary. Extinct species are not known hence.

- Plegadis falcinellus (Linnaeus, 1766)

Known from the Carpathian Basin: Q4/I: Ohábaponor Cave (Bordu Mare-Ohaba Ponor), Romania (Kessler 1985).

- Platalea leucorodia Linnaeus, 1758

Known from the Carpathian Basin: Q4/II: Ecsegfalva 23, Endrőd 39, 119, Szolnok-Szanda, Tiszalúc-Sarkad, Tiszavasvári-Keresztfal (all in Hungary); Parác (Parţa), Romania (Jánossy 1985, Kessler \& Gál 1997, Gál 2004, 2007b, Pike-Tay et al. 2004).

\section{Subord. Ardeae (Wagler, 1831)}

\section{Fam. Ardeidae (Vigors, 1825)}

This family includes herons, egrets, night ravens, the earliest fossil representatives of which are known from the Miocene of the Carpathian Basin (Proardeola walkeri Harrison, 1979) from the middle Miocene of Mátraszőlős, Hungary and Kőalja (Subpiatra), Romania (Gál et al. 2000, Kessler \& Venczel 2009), Egretta polgardiensis Kessler, 2009 from the late Miocene of Polgárdi, Hungary (Kessler 2009a, Ardeidae gen. et sp. indet. from the middle Miocene of Felsőtárkány-Felnémet 2/3, Mátraszőlős 2 (Kessler \& Hír 2012a). Recent species from the Carpathian Basin are known since the Quaternary.

- Egretta alba Linnaeus, 1758

Known from the Carpathian Basin: Q4/II: Ecsegfalva 23, Endrőd 3/6, 119, Esztergom-Alsósziget, Maroslele-Pana, Nagykörü-Tsz, Ószentiván-Tisza-sziget, Tiszaszőlős-Domaháza-puszta (all in Hungary) (Bökönyi 1964, Jánossy 1979c, 1985, Gál 2004, 2007b Pike-Tay et al. 2004).

- Egretta garzetta (Linnaeus, 1766)

Known from the Carpathian Basin: Q4/II: Endröd 3/6; 119, Hungary (Jánossy 1985, Gál 2004, 2007b).

- Ardea cinerea Linnaeus, 1758

Known from the Carpathian Basin: Q4/I: Vindija, Croatia; Puskaporos, Hungary (Lambrecht 1912, 1916, 1933, M. Malez 1961, V. Malez 1973, 1988, 1991, Jánossy 1979c, 1986) Q4/II: Balatonkeresztúr-Réti-dűlö, Balatonszemes-Bagódomb, Ecsegfalva 23, 
Endröd 3/6, 39, 119, Kőtelek-Huszársarok, Maroslele-Pana, Nagykörü-Tsz, Röszke-Lúdvár,Tiszaszőlős-Domaháza-puszta (all in Hungary) (Bökönyi 1964, Jánossy 1979c, 1985, Gál 2004, 2007a, 2007b, Pike-Tay et al. 2004).

- Ardea purpurea Linnaeus, 1766

Known from the Carpathian Basin: Q4/II: Bajcsa Castle, Debrecen-Nyulas, Ecsegfalva 23, Endrőd 39, Mezőfény, Tiszapolgár-Csőszhalom, Polgár-Csőszhalom, Röszke-Lúdvár (all in Hungary) (Jánossy 1979c, 1985, Gál 2002b, 2004, 2007b, Pike-Tay et al. 2004).

- Ardeola sp.

Known from the Carpathian Basin: Q4/I: Vindija, Croatia (M. Malez 1961, V. Malez 1973, 1988, 1991); Puskaporos, Hungary (Kessler, J. 2009b).

- Ixobrychus minutus (Linnaeus, 1866)

Known from the Carpathian Basin: Q1: Betfia 9, Romania (Gál 2002a).

- Botaurus stellaris (Linnaeus, 1758)

Known from the Carpathian Basin: Q4/II: Ecsegfalva 23, Endrőd 3/6 (all in Hungary) (Gál 2004, 2007b, Pike-Tay et al. 2004).

- Botaurus sp.

Known from the Carpathian Basin: Q1: Beremend 17, Hungary (Kessler 2009a).

- Nycticorax nycticorax Linnaeus, 1758

Known from the Carpathian Basin: Q4/II: Ecsegfalva 23, Tiszaszőlös-Domaháza-puszta (all in Hungary) (Gál 2007b, Pike-Tay et al. 2004).

- Nycticorax sp.

Known from the Carpathian Basin: Q1: Beremend 17, Hungary (Kessler, J. 2009a).

\section{Fam. Ciconiidae (Gray, 1840)}

It contains actual storks (Ciconia genus) and tropical storks (Mycteria and Anastomus genera). Their earliest finds in the Carpathian Basin are Ciconia stehlini Jánossy, 1992, from the late Pliocene of Hungarian site Beremend 15 (Jánossy 1996), while Pelargosteon tothi Kretzoi, 1941, was found in the early Pleistocene of Betfia, Transylvania (Romania) (Kretzoi 1962, Gál 2002). Recent species from the Carpathian Basin are known since the Quaternary.

- Ciconia ciconia Linnaeus, 1758

Known from the Carpathian Basin: Q4/II: E

ndröd 39, Lambrecht Cave, Röszke-Lúdvár, Tác-Fövénypuszta, Tác-Gorsium, TúrkeveMóricz (all in Hungary); Püspökfürdö-Lake (Băile Episcopești), Romania (Kessler 1974b, Jánossy 1979c, 1985, Bökönyi 1984, Gál 2004, 2007c).

- Ciconia nigra (Linnaeus, 1758)

Known from the Carpathian Basin: Q4/II: Endröd 39, 119, Szajol-Felsőföld, Tiszalúc-Danka-domb, Kisköre-Szingegát, Röszke-Lúdvár (all in Hungary) (Jánossy 1979c, 1985, Gál 2004, 2007b, 2007c).

- Ciconia sp.

Known from the Carpathian Basin: Q4/II: Endrőd 3/6, Nagykörü-Tsz, Tiszaszőlős-Domaháza-puszta (all in Hungary) (Gál 2004, 2007b).

- + Ciconia stehlini Jánossy, 1992

Known from the Carpathian Basin: MN 16: Beremend 15, Hungary (Jánossy 1992) Q1:

Betfia 9, Romania (Gál 2002) Q2: Beremend 16, 17, Hungary (Jánossy 1996). 
- + Pelargosteon tothi Kretzoi, 1962

Known from the Carpathian Basin: Q1: Köröshegy, Hungary (Kretzoi \& Krolopp 1975) Q2: of Betfia 7 “Aven”, Romania (Kretzoi 1962, Kessler 1975, Gál 2002) Q3/I: of Budapest-Várhegy, Hungary (Jánossy 1980, 1986).

\section{Ord. Anseriformes Wagler, 1831}

Out of the four families of geese, only the Anseridae form a significant group both in terms of the extinct and the recent fauna.

\section{Fam. Anatidae Leach, 1820}

Geese is a species-rich family (with more than 50 recent genera and more than 160 recent species), representatives of which are present on every dry land except Antarctica. Several subfamilies are distinguished within the family.

\section{Subfam. Cygninae (Vigors, 1825)}

Swans are the largest species of the order, living in freshwater areas and seashores. They are herbivores and nest in large groups among aquatic plants. Species living in frigid and temperate zones are migrants. Their extinct species are known from the late Oligocene $(C y g$ nopterus neogradensis Kessler et Hír, 2009 and Cygnanser csakvarensis Lambrecht, 1933 and from the middle Miocene of Hungary (Mátraszőlős and Csákvár) (Kessler \& Hír 2009, respectively Lambrecht 1933). Recent species from the Carpathian Basin are known since the Quaternary from the Carpathian Basin.

- Cygnus olor (Gmelin, 1789)

Known from the Carpathian Basin: Q4/I: Nándori Cave (Curata-Nandru, Romania) (Téglás 1896) Q4/II: Endrőd 3/6, 119, Ludas-Budzsák, Mezőkomárom (all in Hungary); Kazán-szoros-Töröklik (Cuina Turcului Cave-Cazanele), Parác (Parţa) (all in Romania); Starcevo, Serbia (Bökönyi 1974, Kessler 1974, 2009a, Jánossy 1979b, Classon 1980, Kessler \& Gál 1997, Gál 2004, 2007b).

- Cygnus cygnus (Linnaeus, 1758)

Known from the Carpathian Basin: Q4/II: Szilágyzovány (Zăuan), Romania; Starcevo, Serbia (Classon 1980, Gál 2004, Bindea 2008).

- Cygnus sp.

Known from the Carpathian Basin: Q4/II: Dunaújváros-Intrecisa, Hungary; Kazánszoros-Töröklik (Cuina Turcului Cave-Cazanele), Romania (Jánossy 1985, Kessler 1974a).

\section{Subfam. Anserinae Vigors, 1825}

Geese are moderately large herbivores living, nesting, and migrating in large groups. They live near freshwater and seashores, and hatch their eggs in nests on the ground. Branta species of black geese live on wet, grassy areas of the seashore, while Anser species of grey geese live near freshwaters. Tadorna species of shelducks also live on seashores, but rarely near freshwater, and nest in cavities they create. The earliest sign of shelducks comes from the middle Miocene of Hungary (Tadorna minor Kessler et Hír, 2011, from Mátraszőlős) (Kessler \& Hír 2012a). Extinct geese species from the Quaternary of Carpathian Basin are:

- + Anser subanser Jánossy, 1982

Known from the Carpathian Basin: Q3: Somssich-hegy 2, Várhegy, Hungary; Brassó-Fortyogó Hill, (Gensperger Cave-Brașov), Romania (Jánossy 1983). 
Branta species are only known with recent forms since the Pleistocene from the Carpathian Basin. Recent species from the Carpathian Basin are known since the Quaternary. - Anser anser (Linnaeus, 1758)

Known from the Carpathian Basin: Q4/I: Teufelslucken, Austria; Velika Pecina, Croatia; Lambrecht Cave, Mérk (all in Hungary) (Lambrecht 1912, Soergel 1966, Jánossy 1979a, 1979b, V. Malez 1975, 1984, 1988) Q4/II: Teufelslucken, Austria; Balatonkeresztúr-Réti-dűlö, Balatonszemes-Bagódomb, Debrecen-Nyulas, Ecsegfalva 23, Endrőd 3/6, 39, 119, Jászó, Kőtelek, Kőtelek-Huszársarok, Ludas-Budzsák, Mezőfény, Mezőzombor-Községi Cemetery, Nagykörü-Tsz, Szajol-Felsőföld, Szolnok-Szanda, Szerencs-Taktaföldvár, Tatabánya-alsó-Törekvés Cave, Tiszaszőlős-Domaháza-szőlős (all in Hungary); Bólyikő Cave (Piatra Boiului Cave-Lorău), Gyulafehérvár (Alba Iulia), Szegyestel-völgy Caves (Sighiștel Defile), Parác (Parța) (all in Romania); Starcevo, Serbia (Soergel 1966, Bökönyi 1974, Jánossy 1979b, 1985, Classon 1980, Kessler 1982, 1985, 2009a, Kessler \& Gál 1997, Gál 2004, 2005, 2007a, Pike-Tay et al. 2004).

- Anser fabalis Latham, 1790

Known from the Carpathian Basin: Q4/I: Bivak Cave, Hungary; Detrekőszentmiklós-Pálffy Cave (Dzereva Skála), Slovakia (Lambrecht 1913, 1933, Mottl 1938, 1941, Jánossy 1979a, 1979b) Q4/II: Teufelslucken, Austria; Endrőd 39, Maroslele-Pana, Szolnok-Szanda (all in Hungary); Starcevo, Serbia (Bökönyi 1964, Soergel 1966, Jánossy 1979b, 1985, Classon 1980, Gál 2004, 2007b).

- Anser albifrons (Scopoli, 1769)

Known from the Carpathian Basin: Q4/I: Merkenstein, Austria; Remetehegy Niche, Hungary, (Lambrecht 1933, Wettstein \& Mühlhofer 1938) Q4/II: Teufelslucken, Austria; Endrőd 119, Maroslele-Pana, Mezőfény, Ószentiván-Tiszasziget, Pilismarót-Malompatak, Szajol-Felsőföld (all in Hungary); Vársonkolyos Caves (Şuncuiuṣ Defile), Romania (Soergel 1966, Bökönyi 1964, Jánossy 1979b, 1985, Kessler 1985, Gál 2004, 2007b, 2007c).

- Anser erythropus (Linnaeus, 1758)

Known from the Carpathian Basin: Q4/II: Teufelslucken, Austria; Dunaújváros-Intrecisa, Hungary (Soergel 1966, Jánossy 1985).

- Anser sp.

Known from the Carpathian Basin: Q4/I: Pilisszántó, Hungary; Detrekőszentmiklós-Pálffy Cave (Dzereva Skála), Slovakia (Lambrecht 1913, 1933, Mottl 1938, 1941) Q4/II: Ludas-Budzsák, Nagykörü-Tsz, Neszmély-Tekeres Creek, Szegvár-Tüzköves, Esterházy Cave (all in Hungary); Érmihályfalva (Valea lui Mihai), Kisbács-Bácsitorok (Baciu), Körösrév Caves (Vadu Crisului), Vár-Sonkolyos Caves (Şuncuiuṣ Defile) (all in Romania) (Bökönyi 1974, Jánossy 1985, Kessler 1985, 1995, Jurcsák \& Kessler 1986, Gál 2004, 2007b).

- Branta ruficollis (Pallas, 1769)

Known from the Carpathian Basin: Q3: Hundsheim, Austria (Jánossy 1974) Q4/I: Balla Cave, Hungary; Detrekőszentmiklós-Pálffy Cave (Dzereva Skála), Slovakia (Lambrecht 1913, 1933, Mottl 1938, 1941).

- Branta leucopsis (Bechstein, 1803)

Known from the Carpathian Basin: Q4/II: Nagykörü-Tsz, Hungary (Gál 2007b). 


\section{- Branta sp.}

Known from the Carpathian Basin: Q4/II: Parác (Parţa), Romania (Kessler \& Gál 1997, Gál 2004).

- Tadorna tadorna (Linnaeus, 1758)

Known from the Carpathian Basin: Q2: Betfia 5, 7, Romania (Kessler 1975, Gál 2002) Q4/ II: Parác (Parţa), Romania; Padina, Serbia (Classon 1980, Kessler \& Gál 1997, Gál 2004).

- Tadorna ferruginea (Pallas, 1764)

Known from the Carpathian Basin: Q4/I: Teufelslucken, Austria; Körösmart (Râpa), Romania (Soergel 1966, Hamar \& Csák 1969, Kessler 1974).

- Tadorna sp.

Known from the Carpathian Basin: Q2: Nagyharsány-hegy 1-4, Hungary (Jánossy 1979a, 1979b).

\section{Subfam. Anatinae (Vigors, 1825)}

Ducks form the group with the largest number of species, both in terms of recent and fossilized ones. They are the smallest in size of the order, and show wide diversity in their colors and shapes. They are much more attached to water than geese, they feed mostly from waters on invertebrates, but also plants. They usually nest in reed beds, but several species nest in tree burrows. Nestlings are - just like in case of the other families - nidifugous.

Based on their swimming abilities we distinguish between ducks, diving ducks and seaducks, but they undergo numerous methods of classification (Eider Ducks, Stiff-tailed Ducks etc.). Fossil species of their different recent genera are known since the late Oligocene as Mionetta robusta (Milne-Edwards 1868) from Máriahalom, Hungary (Kessler \& Rabi manuscript), and the late Miocene, like Dendronessa sp. from Götzendorf, Austria (Mlíkovský 1991), Mionetta consobrina (Milne-Edwards 1867) from the middle and late Miocene of Hungary (Mátraszőlős) (Kessler \& Hír 2012a), Anas velox (Milne-Edwards 1868) from Hungary (Mátraszőlös and Rudabánya) (Kessler \& Hír 2012a), A. sansanensis (Milne-Edwards 1868) from Austria (Atzelsdorf) (Göhlich 2009), A. denesi Kessler, 2013 from Hungary (Polgárdi) (Kessler, J. 2013), A. albae Jánossy, 1979 from the late Miocene and Pliocene of Hungary (Polgárdi and Csarnóta 2, Villány 3) (Jánossy 1979b, 1991) and from the middle Miocene of Romania (Kőalja-Subpiatra) (Kessler \& Venczel 2009), Clangula matraensis and Mergus minor Kessler, 2009 from the middle Miocene of Mátraszőlős, Hungary (Kessler, J. 2009a), Bucephala cereti Boeuf et Mourer-Chauviré, 1992 from Mátraszőlős, Hungary (Gál et al. 1998-1999), Mergus minor Kessler, 2009 from Mátraszőlős, Hungary (Kessler, J. 2009a) and Mergus connectens Jánossy, 1972 from the early Pleistocene of Betfia, Romania (Jánossy 1979b).

Recent species from the Carpathian Basin are known since the Quaternary. - Anas plathyrhynchos Linnaeus, 1758

Known from the Carpathian Basin: Q3: Vértesszőlős 2, Hungary (Jánossy, 1969) Q4/I: Merkenstein, Teufelslucken (all in Austria); Vindija, Croatia; Bajót-Jankovich Cave, Budapest-Remetehegy Niche, Cserépfalu-Subalyuk Cave, Csákvár-Eszterházy Cave, Herman Ottó Cave, Pilisszántó Niche, Puskaporos Niche, Répáshuta-Balla Cave, Szilvásvárad-Istállóskői Cave, Tokod-Nagyberek, Varbó-Lambrecht Kálmán Cave (all in Hungary); Körösmart (Râpa), Nándori Cave (Curata-Nandru), Ohábaponor (Bordu Mare Cave-Ohaba Ponor), Körösrév-Kecske Cave, Pince Cave (Caprei and Pivniţe Caves-Vadu Crișului), 
(all in Romania); Detrekőszentmiklós-Pálffy Cave (Dzereva Skála), Slovakia (Lambrecht 1912, 1913, 1915, 1916, 1933, Mottl 1938, 1941, Wettstein \& Mühlhofer 1938, M. Malez 1961, Jánossy 1965, 1979a, 1979b, 1986, Soergel 1966, V. Malez 1973, 1988, 1991, Kessler 1974a, 1985, Fischer \& Stephan 1977, Jurcsák \& Kessler 1988, Gál 2002a, 2003) Q4/ II: Grosse Offenbergerhöhle, Teufelslucken (all in Austria); Bodajk-Rigólyuk, Csákvár-Esterházy Cave, Dunaújváros-Intrecisa, Ecsegfalva 23, Endrőd 3/6, 39, 119, Legény Cave, Maroslele-Pana, Paks-Dunakömlőd, Röszke-Ludvár, Szajol-Felsőföld, Szolnok-Szanda, Tác-Fövénypuszta, Tác-Gorsium, Tiszaszőlős-Domaháza-puszta (all in Hungary); Kisderzsida (Đerșida Mică), Kőrösbánlak Cave (Bălnaca), Kőrösrévi Caves (Vadu Crișului Defile), Ompolymező (Poiana Ampoiului), Parác (Parţa), Peterd-Tordai-hasadék-Magyar Cave (Cheile Turzii Defile-Ungureasca Cave-Petrești), Püspökfürdő Lake (Băile Episcopești), Remetelórév-Bólyikő Cave (Piatra Boiului-Lorău) (all in Romania) (Lambrecht 1914, Bökönyi 1964, 1984, Soergel 1966, Kessler 1974b, 1982, 1985, Jánossy 1979a, 1979b, 1985, Kordos 1984, Körösi 1991, Bocheński \& Tomek 1994, Kessler \& Gál 1997, 1998, Bindea 2008, Gál 2004, 2007b, 2007c, Pike-Tay et al. 2004).

- Anas penelope Linnaeus, 1758

Known from the Carpathian Basin: Q2: Nagyharsány-hegy 1-4, Hungary (Lambrecht 1916, 1933, Jánossy 1979a) Q4/I: of Budapest-Remetehegy Niche, Puskaporos Niche, Répáshuta-Balla Cave (all in Hungary); Körösmart (Râpa), Nándori Cave (Curata-Nandru), Ohábaponor (Bordu Mare Cave-Ohaba Ponor) (all in Romania); Detrekőszentmiklós-Pálffy Cave (Dzereva Skála-Pálfy Cave), Slovakia (Lambrecht 1912, 1913, 1916, 1933, Mottl 1938, 1941, Kessler 1974, 1985, Fischer \& Stephan 1977, Jánossy 1979a, 1986, Musil 1980, Jurcsák \& Kessler 1988, Gál 2002a, 2003) Q4/II: Teufelslucken, Austria; Dunaújváros-Intrecisa, Endrőd 39, Tiszaszőlős-Domaháza-puszta (all in Hungary); Püspökfürdő Lake (Băile Episcopești), Romania (Jánossy 1965, 1985, Soergel 1966, Kessler 1974, 1985, Gál 2004, 2007b). - Anas strepera Linnaeus, 1758

Known from the Carpathian Basin: Q1: Beremend 17, Hungary; Betfia 2, Körösmart (Râpa), (all in Romania) (Kormos 1913, Čapek 1917, Lambrecht 1933, Kretzoi 1941, Kessler 1974a, 1975, Jánossy 1977, 1979b, 1992, 1996, Gál 2002a) Q4/I: Pilisszántói Niche, Hungary; Detrekőszentmiklós-Pálffy Cave (Dzereva Skála), Slovakia (Lambrecht 1913, 1915, 1933, Mottl 1938, 1941, Jánossy 1979a, 1979b 1986) Q4/II: Ecsegfalva 23, Hungary (Pike-Tay et al. 2004, Gál 2007b).

- Anas crecca Linnaeus, 1758

Known from the Carpathian Basin: Q3/II: Vindija, Croatia (M. Malez 1961, V. Malez 1973, 1988, 1991) Q4/I: Velika Pecina, Vindija (all in Croatia); Bajót-Hóman Cave, Budapest-Remetehegy Niche, Puskaporos Niche, Tatabánya-Szelim Cave (all in Hungary); Detrekőszentmiklós-Pálffy Cave (Dzeráva Skála-Plavecky Mikulas), Novi III (Novi Caves) (all in Slovakia) (Nehring 1880, Lambrecht 1912, 1913, 1916, 1933, Mottl 1938, 1941, M. Malez 1961, V. Malez 1973, 1975, 1984, 1988, 1991, Jánossy 1979a, 1979b, 1986) Q4/II: Teufelslucken, Austria; Bodajk-Rigólyuk, Csákvár-Esterházy Cave, Ecsegfalva 23, Endrőd 3/6, Tác-Fövénypuszta, Tác-Gorsium (all in Hungary); Diószeg (Diosig), Gura Chindiei (all in Romania) (Soergel 1966, Kessler 1977, 1985, Jánossy 1979b, 1985, Bökönyi 1984, Kordos 1984, Gál 2004, 2007b, Pike-Tay et al. 2004). 
- Anas acuta Linnaeus, 1758

Known from the Carpathian Basin: Q1: Beremend 17, Hungary; Betfia 2, Romania (Kormos 1913, Čapek 1917, Lambrecht 1933, Kretzoi 1941, Kessler 1975, Jánossy 1977, 1979b, 1992, 1996, Gál 2002) Q2: Nagyharsány-hegy 1-4, Hungary (Lambrecht 1916, 1933, Jánossy 1979a, 1979b) Q3: Vértesszőlős, Hungary (Jánossy 1979a, 1979b, 1990) Q4/I: Vindija, Croatia; Detrekőszentmiklós-Pálffy Cave (Dzeráva Skála-Plavecky Mikulas), Slovakia (Lambrecht 1913, 1933, Mottl 1938, 1941, M. Malez 1961, V. Malez 1973, 1988, 1991) Q4/II: Teufelslucken, Austria; Ecsegfalva 23, Hungary; Peterd-Tordai-hasadék-Magyar Cave (Cheile Turzii Defile-Petrești), Püspökfürdő Lake (Băile Episcopești) (all in Romania) (Soergel 1966, Kessler 1974, 1985, Kessler \& Gál 1998, Gál 2004, 2007b, Pike-Tay et al. 2004).

- Anas querquedula Linnaeus, 1758

Known from the Carpathian Basin: Q1-2: Nagyharsány-hegy 1-4, Hungary; Betfia 2, 9, Betfia “Aven" (all in Romania) (Kormos 1913, Lambrecht 1916, 1933, Čapek 1917, Kretzoi 1941, Kessler 1975, Jánossy 1977, 1979b, Gál 2002a) Q4/I: Vindija, Croatia; Cserépfalu-Subalyuk Cave, Pilisszántó Niche, Tokod-Nagyberek (all in Hungary); Körösmart (Râpa), Romania (Lambrecht 1915, 1933, M. Malez 1961, V. Malez 1973, 1988, 1991, Kessler 1974, Jánossy 1979a, 1979b, Gál 2002) Q4/II: Teufelslucken, Austria; Ecsegfalva 23, Endrőd 3/6, Legény Cave (all in Hungary); Gálospetri (Galoṣpetru), Körösbánlak Cave (Bălnaca), Püspökfürdö Lake (Băile Episcopești) (all in Romania) (Lambrecht 1914, Soergel 1966, Kessler 1974, 1982, 1985, Jánossy 1985, Gál 2002a, 2004, 2007b, Pike-Tay et al. 2004).

- Anas clypeata (Linnaeus, 1758)

Known from the Carpathian Basin: Q1-2: Beremend 16, 17, Nagyharsány-hegy 1-4 (all in Hungary); Betfia 2, 9, Betfia “Aven" (all in Romania) (Lambrecht 1916, 1933, Kessler 1975, Jánossy 1979a, 1979b, 1992, 1996, Gál 2002a) Q3: Süttő, Hungary (Jánossy 1979a) Q4/I: Körösmart (Râpa), Nándori Cave (Curata-Nandru) (all in Romania) (Jánossy 1965, 1979a, 1979b, Kessler 1974a, Fischer \& Stephan 1977, Jurcsák \& Kessler 1988, 1985, Gál 2002a, 2003) Q4/II: Gálospetri (Galoṣpetreu), Körösrévi Caves (Vadu Crișului Defile), Püspökfürdő Lake (Băile Episcopești), Vársonkolyosi Caves (Şuncuiuṣ Defile) (all in Romania); Starcevo, Serbia; Endrőd 3/6, 119, Hungary (Jurcsák 1969, Kessler 1974b, 1980-1981, 1982, 1985, Classon 1980, Jánossy 1985, Gál 2002a, 2004, 2007b).

- Anas sp.

Known from the Carpathian Basin: Q1: Beremend 17, Hungary; Betfia 2, Romania (Kormos 1913, Čapek 1917, Lambrecht 1933, Kretzoi 1941, Kessler 1975, 2009a, Jánossy 1977, 1979b, Gál 2002a) Q3/I: Vindija, Croatia; Budapest-Vár-Hill, Hungary (M. Malez 1961, V. Malez 1973, 1988, 1991, Jánossy 1979a, 1979b) Q4/I: Krapina, Croatia; Tata, Hungary; Nándori Cave (Curata-Nandru), Romania; Lándzsásötfalu (Hŏrka-Ondrej), Liszkófalva-Baráthegyi Cave (Lisková), Novi III, (Novi Caves) (all in Slovakia) (Lóczy 1877, Nehring 1880, Téglás 1880, Róth 1881, Lambrecht 1912, 1915, 1933, V. Malez 1973, 1984, V. Malez-Bačić 1975, Jánossy 1979b, 1986, M. Malez \& V. Malez 1988, Mlíkovskỳ 2000c) Q4/II: Berettyószentmárton, Endrőd 3/6, Maroslele-Pana, Mezőfény, Pomáz-Zravlyák, Szajol-Felsőföld, Szolnok-Szanda, Tiszalúc-Sarkad (all in Hungary); Radnót (Iernuţ), Romania (Bökonyi 1964, Jánossy 1977, 1979b, 1985, Gál 2004, 2007b, 2007c, Bindea 2008). 
- Ayhthya nyroca (Güldenstadt, 1769)

Known from the Carpathian Basin: Q1: Betfia 2, 9, Romania (Kormos 1913, Čapek 1917, Lambrecht 1933, Kretzoi 1941, Kessler 1975, Jánossy 1977, 1979b, Gál 2002a) Q3/I: Tarkő 3, Hungary (Jánossy 1962, 1976, 1979a, 1979b) Q4/I: Vindija, Croatia; Bajót-Jankovich Cave, Budapest-Remetehegy Niche, Csákvár-Eszterházy Cave, Pilisszántói I. Niche, Puskaporos Niche (all in Hungary); Nándori Cave (Curata-Nandru), Romania (Lambrecht 1912, 1915, 1916, 1933, Mottl 1941, M. Malez 1961, Jánossy 1965, 1979a, 1979b, 1986, V. Malez 1973, 1988, 1991, Fischer \& Stephan 1977, Kessler 1985, Jurcsák \& Kessler 1988, Gál 2002a, 2003) Q4/II: Teufelslucken, Austria; Ecsegfalva 23, Endrőd 119, Legény Cave, Ludas-Budzsák, Mezőfény, Nagykörü-Tsz, Röszke-Ludvár, Tiszaszőlös-Domaháza-puszta (all in Hungary) (Lambrecht 1914, Soergel 1966, Bökönyi 1974, Jánossy 1985, Gál 2004, 2007b, Pike-Tay et al. 2004).

- Aythya fuligula Linnaeus, 1758

Known from the Carpathian Basin: Q4/I: Krapina, Vindija (all in Croatia); Detreköszentmiklós-Pálffy Cave (Dzeráv a Skála-Plavecky Mikulas), Slovakia (Lambrecht 1913, 1915, 1933, Mottl 1938, 1941, M. Malez 1973, 1984, 1988, 1991, Malez-Bačić 1975, M. Malez \& V. Malez 1988) Q4/II: Ecsegfalva 23, Felsötárkány-Petényi Cave, Ludas-Budzsák, Röszke-Ludvár, Tác-Gorsium, Tápiószele-Tüzköves (all in Hungary); Gálospetri (Galoṣpetreu), Romania (Bökönyi 1974, 1984, Jánossy 1977, 1979a, 1979b, 1985, Kessler 1980-1981, Gál 2002a, 2004, 2007b, Pike-Tay et al. 2004).

- Aythya ferina (Linnaeus, 1758)

Known from the Carpathian Basin: Q4/I: Teufelslucken, Austria; Vindija, Croatia; Hámor-Puskaporos Niche, Hungary (Lambrecht 1912, 1916, 1933, Mottl 1941, M. Malez 1961, Soergel 1966, V. Malez 1973, 1988, 1991, Jánossy 1979a, 1979b, 1986) Q4/II: Ecsegfalva 23, Endrőd 3/6, 119, Maroslele-Pana, Nagykörü-Tsz, Tác-Gorsium, Tápiószele-Tüzköves (all in Hungary) (Bökönyi 1964, 1984, Jánossy 1977, 1979b, 1985, Gál 2004, 2007b, Pike-Tay et al. 2004).

- Aythya marila (Linnaeus, 1761)

Known from the Carpathian Basin: Q4/II: Tiszaszőlös-Domaháza-puszta, Hungary (Gál 2007b).

- Aythya sp.

Known from the Carpathian Basin: Q1: Beremend 17, Hungary (Kessler 2009a) Q2: Nagyharsány-hegy, Somssich-hegy 2 (all in Hungary) (Jánossy 1981a, 1982b, 1983, 1986, Kessler 2009a).

- Bucephala clangula (Linnaeus, 1758)

Known from the Carpathian Basin: Q4/I: Bajót-Hóman Cave, Hungary (Jánossy 1979a, 1979b) Q4/II: Gura Chindiei, Romania (Kessler 1985).

- Melanitta nigra (Linnaeus, 1758)

Known from the Carpathian Basin: Q4/I: Detrekőszentmiklós-Pálffy Cave (Dzeráva Skála-Plavecky Mikulas), Slovakia (Lambrecht 1913, 1933).

- Mergus albellus Linnaeus, 1758

Known from the Carpathian Basin: Q4/I: Jankovits Cave, Puskaporos (all in Hungary); Detrekőszentmiklós-Pálffy Cave (Dzeráva Skála-Plavecky Mikulas), Slovakia (Lambrecht 1913, 1933, Jánossy 1986). 
- Mergus serrator Linnaeus, 1758

Known from the Carpathian Basin: Q4/II: Kazán-szoros-Töröklik (Cuina Turcului Cave-Cazanele), Vársonkolyos-Kismagyar Cave (Napișteleu Cave, Şuncuiuṣ) (all in Romania); Detrekőszentmiklós-Pálffy Cave (Dzeráva Skála-Plavecky Mikulas), Slovakia (Lambrecht 1913, 1933, Kessler 1974c, 1977a, 1983, 2009a).

- Mergus merganser Linnaeus, 1758

Known from the Carpathian Basin: Q4/I: Vindija, Croatia (M. Malez 1961, V. Malez 1973, 1988, 1991); Balla Cave, Hungary (Lambrecht 1912) Q4/II: Teufelslucken, Austria; Kazán-szoros-Töröklik (Cuina Turcului Cave-Cazanele), Ohábaponor (Bordu Mare Cave, Ohaba Ponor) (all in Romania) (Soergel 1966, Kessler 1981, 1985, 2009a).

- Anatidae indet.

Known from the Carpathian Basin: Q1: Betfia 9, Romania (Gál 2002); Q2: Somssich-hegy 2, Hungary (Jánossy 1981a, 1982b, 1983, 1986).

\section{Subord. Ralli (Reichenbach, 1852)}

This suborder is containing rails, finfoots and members of an extinct family.

\section{Fam. Rallidae Vigors, 1825}

Rails are small to medium-sized birds which prefer hiding, and live in wet environments with tall grass. There are also flightless and barely flying species, especially on islands of Oceania. Most well-known are coots, rails, corncrakes and crakes. From the Upper Oligocene of Kolozsvár (Cetățuie, Cluj, Romania), we known Rallicrex kolozsvarensis Lambrecht, 1933 (Lambrecht 1933), from the early, middle and late Miocene of Hungary (Litke, Felsőtárkány, Mátraszőlős, Polgárdi) R. litkensis Kessler et Hír, 2012 and R. polgardiensis Jánossy, 1991 (Jánossy 1991, Kessler 2009b, Kessler \& Hír 2012b). Several species are known from the Miocene of Europe, including Hungary, such as Paleoaramides beaumontii (Milne-Edwards 1869) from early Pleistocene of Beremend 17, Miorallus major (Milne-Edwards 1869) from late Miocene of Rudabánya, Porzana matraensis Kessler, 2009 from middle Miocene of Mátraszőlős, P. estramosi Jánossy, 1979 and P. kretzoii Kessler, 2009 from middle and late Miocene of Mátraszőlős and Polgárdi (Jánossy 1979 a, 1979b, 1991, Gál et al. 1998-1999, Kessler, J. 2009b), Rallidae indet. from middle Miocene of Kőalja (Subpiatra, Romania) (Kessler \& Venczel 2009).

Recent species from the Carpathian Basin are known since the Quaternary.

- Porzana porzana (Linnaeus, 1766)

Known from the Carpathian Basin: Q1: Beremend 16, 17, Hungary; Betfia 9, Romania (Jánossy 1992, 1996, Gál 2002) Q4/I: Budapest-Remetehegyi Niche, Hámor-Puskaporos Niche, Pilisszántói I. Niche, Szilvásvárad-Istállóskői Cave, Tatabánya-Szelim Cave (all in Hungary); Barcarozsnyó (Rîșnov), Romania; Detrekőszentmiklós-Pálffy Cave (Dzeráva Skála-Plavecky Mikulas), Slovakia (Lambrecht 1912, 1915, 1916, 1933, Jánossy 1952, 1955, 1979a, 1979b, Gál 1998, 2002) Q4/II: Bodajk-Rigólyuk, Ecsegfalva 23 (all in Hungary) (Kordos 1984, Pike-Tay et al. 2004, Gál 2007b).

- Porzana parva (Scopoli, 1769)

Known from the Carpathian Basin: Q1: Beremend 17, Hungary; Betfia 9, Romania (Jánossy 1992, 1996, Gál 2002). 
- Porzana pusilla Pallas, 1776

Known from the Carpathian Basin: Q1: Betfia 9, Romania (Gál 2002).

- Crex crex Linnaeus, 1758

Known from the Carpathian Basin: Q1: Betfia 2, 9, Romania (Kormos 1913, Čapek 1917, Lambrecht 1933, Kessler 1975, Jánossy 1979b, Gál 2002a) Q4/I: Teufelslucken, Austria; Bajót-Hóman Cave, Budapest-Remetehegyi Niche, Felsőtárkány-Peskő Cave, Gencsapáti, Hámor-Puskaporos Niche, Kőszeg, Pilisszántói I. Niche, Répáshuta-Balla Cave, Szilvásvárad-Istállóskői Cave, Tatabánya-Kálvária Hill No. 4. Cave, Tatabánya-Szelim Cave, Varbó-Lambrecht Kálmán Cave (all in Hungary); Barcarozsnyó (Rîșnov), Körösmart (Râpa), Nándori Cave (Curata-Nandru) (all in Romania); Detrekőszentmiklós-Pálffy Cave (Dzeráva Skála-Plavecky Mikulas), Slovakia (Lambrecht 1912, 1915, 1916, 1933, Jánossy 1952, 1955, 1979a, 1979b, Soergel 1966, Hamar \& Csák 1969, Kessler 1974, Jurcsák \& Kessler 1988, 2003, Gál 1998, 2002a, 2004, 2005) Q4/II: Knochenhöhle, Austria; Felsőtárkány-Petényi Cave, Legény Cave (all in Hungary); Vársonkolyosi Caves (Şuncuiuș Defile), Romania (Lambrecht 1914, Jánossy 1979a,c, Kessler 1982, Bocheński \& Tomek 1994).

- Crex sp.

Known from the Carpathian Basin: Q1: Beremend 16, 17, Hungary (Jánossy 1992, 1996). - Rallus aquaticus Linneaus, 1758

Known from the Carpathian Basin: Q1: Betfia 2, 9, Romania (Kormos 1913, Čapek 1917, Lambrecht 1933, Kessler 1975, Jánossy 1979b, Gál 2002) Q4/I: Merkenstein, Austria; Vindija, Croatia; Bajót-Jankovich Cave, Budapest-Remetehegyi Niche, Cserépfalu-Subalyuk Cave, Felsőtárkány-Peskő Cave, Gencsapáti, Hámor-Puskaporos Niche, Pilisszántói I. Niche, Tatabánya-Szelim Cave, Tokod-Nagyberek, Varbó-Lambrecht Kálmán Cave (all in Hungary); Körösmart (Râpa), Romania; Detreköszentmiklós-Pálffy Cave (Dzeráva Skála-Plavecky Mikulas), Slovakia (Lambrecht 1912, 1915, 1916, 1933, Wettstein \& Mühlhofer 1938, M. Malez 1961, Hamar \& Csák 1969, V. Malez 1973, 1988, 1991, Kessler 1974a, Jánossy 1979a, 1979b, Gál 2002a) Q4/II: Bodajk-Rigólyuk, Cserépfalu-Subalyuk (all in Hungary) (Kordos 1984, Kessler 2009b).

- Gallinula chloropus (Linneaus, 1758)

Known from the Carpathian Basin: Q1: Beremend 17, Hungary (Jánossy 1992, 1996) Q4/I: Vindija, Croatia (M. Malez 1961, V. Malez 1973, 1988, 1991) Q4/II: Grosse Offenbergerhöhle, Austria; Ecsegfalva 23, Mezőlak-Szélmező Puszta, Tiszaszőlős-Domaháza puszta (all in Hungary); Vársonkolyos-Kismagyar Cave (Napișteleu-Şuncuiuṣ), Romania (Kessler 1977, Krolopp \& Vörös 1982, Bocheński \& Tomek 1994, Gál 2002a, 2007b, Pike-Tay et al. 2004).

- Fulica atra Linnaeus, 1758

Known from the Carpathian Basin: Q2: Betfia 5, Romania (Kessler 1975, Jánossy 1979b, Gál 2002) Q4/II: Teufelslucken, Austria; Bajcsa-Vár, Ecsegfalva 23, Endrőd 3/6, 39,119, Maroslele-Pana, Mezőfény, Mezőlak-Szélmezö-Puszta, Mezőzombor-községi cemetery, Nagykörü-Tsz, Ordacsehi-Kistöltés, Ószentiván-Tiszasziget, Tiszaszőlős-Domaháza-puszta, Tiszavalk-Négyesi határ, Visegrád Castle (all in Hungary); Gálospetri (Galoṣpetreu), Püspökfürdő Lake (Băile Episcopești) (all in Romania) (Bökönyi 1964, Bökönyi \& Jánossy 
1965, Soergel 1966, Kessler 1974b, 1980-1981, 1985, Jánossy 1979b, 1985, Krolopp \& Vörös 1982, Gál 2002a, 2004, 2005, 2007b, Pike-Tay et al. 2004).

\section{Fam. Heliornithidae (Gray, 1849)}

Finfoots resemble smaller grebes in appearance and lifestyle quite closely. They live in South America and Africa (only 5 recent species in total). Only one fossil find is known, namely Heliornis sumegensis Kessler, 2009 from the late Miocene of Hungary (Sümeg) (Kessler, J. 2009b).

\section{Subord. Grues Bonaparte, 1854}

Similarly to the previous suborder, they are fairly large birds (with long necks and legs) not tied too much to wet and aquatic environments. They are excellent flyers, with numerous extinct and recent taxons. They nest on the ground and their nestlings are nidifugous. They are usually herbivores, but there are also omnivorous and carnivorous types.

\section{Fam. Gruidae (Vigors, 1825)}

Cranes represent larger species of the order due to their excessively long necks and legs. They are present on all continents except Antarctica and Australia. They live on tundras, forest-grass areas and high plateaus. They feed on plants, smaller vertebrates and insects. They build their nests on the ground, their nestlings are nidifugous. They are migratorious. Extinct species Balearica excelsa Milne-Edwards, 1868 is known from the late Oligocene of Hungary (Máriahalom) (Kessler \& Rabi manuscript), while extinct species of the genus Grus (G. pentelici Gaudry, 1862) are known from the late Miocene of Hungary (Csákvár) (Kretzoi 1957).

Recent species from the Carpathian Basin are known since the Quaternary.

- Grus grus (Linnaeus, 1758)

Known from the Carpathian Basin: Q4/I: Teufelslucken, Austria; Lovas, Hungary; Körösmart (Râpa), Romania (Soergel 1966, Hamar \& Csák 1969, Kessler 1974a, Jánossy 1979b, 1987, Gál 2002a) Q4/II: Alattyán-Tulát, Balatonkeresztúr-Réti-dűlő, Békés-Városerdő, Dunaújváros-Koszider, Ecsegfalva 23, Endrőd 39,119, Folyás-Szilmeg, Füzesabony, Gyula-Castle, Jánoshida, Kőtelek-Huszársarok, Ludas-Budzsák, Maroslele-Pana, Polgár-Folyás, Röszke-Ludvár, Székesfehérvár, Szolnok-Szanda, Tác-Fövénypuszta, Tác-Gorsium, Tiszalúc-Dankadomb, Tiszalúc-Sarkad (all in Hungary); Aranyosmeggyes (Medieșu Auriu), Kisbács-Bácsitorok (Baciu), Parác (Parţa), Peterd-Tordai-hasadék-Magyar Cave (Cheile Turzii Defile Caves-Petrești), Szalacs (Sălacea), Szilágyzovány (Zăuan) (all in Romania); Starcevo, Serbia; Tokod-Erzsébetakna, Hungary (Bökönyi 1964, 1974, 1984, Bökönyi \& Jánossy 1965, Jánossy 1979a, 1979b, 1985, Jurcsák \& Kessler 1988, Kessler 1995, Kessler \& Gál 1997, 1998, Bartosiewicz 1997, Classon 1980, Gál 2004, 2005, 2007b, 2007c, Bindea 2008, Pike-Tay et al. 2004).

\section{Subord. Otides Wagler, 1830}

Fam. Otididae (Gray, 1840)

Bustards are large birds, yet they fly well. They live in Eurasia, Africa and Australia. They live in areas with tall grass. They feed on plants and invertebrates. Sexual dimorphism is significant; the males keep a harem, they nest on the ground, their nestlings are nidifugous. Certain species migrate. Extinct species known from the Qarternary of the Carpathian Basin are the following: 
- Otis khozatzkii Bochenski et Kurchkin, 1987

Known from the Carpathian Basin: MN 13: Polgárdi 4, 5, Hungary (Jánossy 1991).

- + Otis kalmani Jánossy, 1972

Known from the Carpathian Basin: MN 13: Polgárdi 4,5, Hungary (Kessler 2009b) MN 15: Beremend 26, Csarnóta 2 (all in Hungary) (Kessler 2009b) MN 16: Beremend 38, Hungary (Kessler 2009b) Q1: Beremend 16, 17, Hungary; Betfia 2, 9, Romania (Kessler 1975, Jánossy 1979c, 1992, 1996, Gál 2002a) Q2: Nagyharsány-hegy 1-4, Villány 5 (all in Hungary); Betfia 5, 7, Romania (Jánossy 1979c, Gál 2002a, Kessler 2009b).

- + Otis lambrechti Kretzoi, 1941

Known from the Carpathian Basin: MN 15: Beremend 26, Hungary (Kessler 2009b) MN 16: Beremend 38, Hungary (Kessler 2009b) Q1: Beremend 17, Hungary; Betfia 2, 9 Romania (Kretzoi 1941, 1962, Kessler 1975, Jánossy 1979c, 1992, 1996, Gál 2002a) Q2: Nagyharsányhegy 1-4, Hungary; Betfia 5, 7, Romania (Kretzoi 1941, 1962, Kessler 1975, Jánossy 1979a,c, Gál 2002a) Q3: Hundsheim, Austria; Beremend 23, Hungary; Betfia 7, Romania (Jánossy 1974, 1979c, Kessler 1975, 2009b, Gál 2002a).

Recent species from the Carpathian Basin are known since the Quaternary.

- Otis tarda Linnaeus, 1758

Known from the Carpathian Basin: Q3/I: Budapest-Várhegy (Jánossy 1979c) Q4/I: Varbó-Lambrecht Kálmán Cave (Jánossy 1979a,c, 1986) Q4/II: Teufelslucken, Austria; Ecsegfalva 23, Endröd 39, 119, Ludas-Budzsák, Nosza-Gyöngypart, Szajol-Felsőföld, Tószeg-Laposhalom, Turkeve-Móricz (all in Hungary); Nagyvárad-Szálka Hill (Salca-Oradea), Parác (Parţa), Peterd-Tordai-hasadék-Magyar Cave (Cheile Turzii Defile, Ungureasca Cave-Petrești), Vizakna (Ocna Sibiului) (all in Romania); Starcevo, Szabadka-Palics (Palič-Subotica) (all in Serbia); Érsekújvár (Nové Zámsky) Kisvárad (Nitriansky Hrádok) (all in Slovakia) (Soergel 1966, Jurcsák \& Kessler 1973, Bökönyi 1974, Jánossy 1979c, 1985, Kessler \& Gál 1997, 1998, Classon 1980, Gál 2004, 2007b, 2007c, Pike-Tay et al. 2004, Bindea 2008).

- Otis tetrax Linnaeus, 1758

Known from the Carpathian Basin: Q3/I: Budapest-Várhegy, Hungary (Jánossy 1979a,c) Q4/I: Subalyuk, Hungary (Jánossy 1979a,c) Q4/II: Tác-Fövény-Puszta, Tác-Gorsium (all in Hungary) (Jánossy 1979c, 1985, Bökönyi 1984).

- Otis sp.

Known from the Carpathian Basin: Q2: Somssich-hegy 2, Hungary (Jánossy 1981a, 1982b, 1983, 1986) Q3/I: Betfia 7/4, Romania (Kessler 1975, Jánossy 1979c, Gál 2002a).

\section{Ord. Charadriiformes (Huxley, 1867)}

Charadriiformes is an order rich in species which are small to middle in size, and prefer aquatic environments. They feed on invertebrates and smaller vertebrates, or can even be necrophages (like seagulls, for example). They live in pairs and hatch their eggs on the ground or ledges of cliffs. Their nestlings are nidifugous. They might migrate in frigid and temperate zones. They occur worldwide. Current-day avian fauna is represented by stone curlews, avocets, oystercatchers, plowers, redshanks, sandpipers, jacanas, quails, pranticoles, razorbills, skuas, gulls, arctic terns, marsh terns etc. Their earliest find in Europe is Kievornis rogovitshi Averianov, Potapova et Nessov, 1990 from the Eocene of Ukraine. 


\section{Subord. Charadrii (Huxley, 1867) \\ Fam. Scolopacidae (Vigors, 1825)}

Sandpipers are the species-rich family of woodcocks, snipes, curlews, godwits, redshanks, phalaropes, etc. They live in wet environments and feed on invertebrates. They nest on the ground and are excellent flyers. They are migrants. Extinct species have already been reported from the Miocene and Pliocene of Carpathian Basin sites. (For example Gallinago veterior Jánossy, 1979 from the middle and late Miocene and Pliocene of Hungary (Mátraszőlös, Polgárdi, Csarnóta). Other extinct species is Scolopax baranensis Jánossy, 1979, is known from the middle and late Pliocene of Hungary (Csarnóta, Beremend) (Jánossy 1979b, Gál et al. 1998-1999, Kessler, J. 2009b).

Recent species from the Carpathian Basin are known since the Quaternary.

- Gallinago media (Latham, 1787)

Known from the Carpathian Basin: Q1: Betfia 2, Romania (Kormos 1913, Čapek 1917, Lambrecht 1933, Kretzoi 1941, Kessler 1975, Jánossy 1979b, Gál 2002) Q3/I: Tarkő 11, Hungary (Jánossy 1979a, 1979b) Q4/I: Grosse Offenbergerhöhle, Austria; Körösmart (Râpa), Szegyestel-Tibocoaia Cave (Sighiștel Defile-Tibocoaia Cave) (all in Romania); Detrekőszentmiklós-Pálffy Cave (Dzeráva Skála-Plavecky Mikulas), Slovakia; Hámor-Puskaporos Niche, Pilisszántói I. Niche (all in Hungary) (Lambrecht 1912, 1913, 1916, 1933, Hamar \& Csák 1969, Kessler 1974, 1982, 1985, Jánossy 1979a, 1979b, 1986, Bocheński \& Tomek 1994, Gál 2002a).

- Gallinago gallinago Linnaeus, 1758

Known from the Carpathian Basin: Q1: Betfia 2, Romania (Kormos 1913, Čapek 1917, Lambrecht 1933, Kretzoi 1941, Kessler 1975, Jánossy 1977, 1979a, 1979b, Gál 2002a) Q4/I: Vindija, Croatia; Bajót-Hóman Cave, Hámor-Puskaporos Niche, Pilisszántói I. Niche (all in Hungary); Detrekőszentmiklós-Pálffy Cave (Dzeráva Skála-Plavecky Mikulas), Slovakia (Lambrecht 1912, 1913, 1915, 1916, 1933, M. Malez 1961, V. Malez 1973, 1988, 1991, Jánossy 1979a, 1979b, 1986) Q4/II: Gálospetri (Galoṣpetreu), Romania (Kessler 1980-1981, Gál 2002a).

- Lymnocryptes minimus (Brünnich, 1764)

Known from the Carpathian Basin: Q4/II: Grosse Offenbergerhöhle, Austria (Bocheński \& Tomek 1994).

- Limosa limosa (Linnaeus, 1758)

Known from the Carpathian Basin: Q1: Betfia 2, 9, Romania; Beremend 17, Hungary (Kormos 1913, Čapek 1917, Lambrecht 1933, Kretzoi 1941, Kessler 1975, Jánossy 1977, 1979a, 1979b, 1992, 1996; Gál 2002a) Q1-2: Betfia “Aven”, Romania (Kessler 1975, Jánossy 1979b, Gál 2002a) Q4/I: Vindija, Croatia; Hámor-Puskaporos Niche, Hungary; Detrekőszentmiklós-Pálffy Cave (Dzeráva Skála-Plavecky Mikulas), Slovakia (Lambrecht 1912, 1913, 1916, 1933, M. Malez 1961, V. Malez 1973, 1988, 1991, Jánossy 1979a, 1979b, 1986) Q4/II: Ecsegfalva 23, Hungary (Pike-Tay et al. 2004, Gál 2007b).

- Numenius arquata (Linnaeus, 1758)

Known from the Carpathian Basin: Q4/I: Cserépfalu-Subalyuk Cave, Gencsapáti, Tata, Tatabánya-Szelim Cave (all in Hungary); Detrekőszentmiklós-Pálffy Cave (Dzeráva Skála-Plavecky Mikulas), Slovakia (Lambrecht 1913, 1915, 1933, Jánossy 1979a, 1979b, 1986) 
Q4/II: Starcevo, Serbia (Classon 1980, Gál 2004).

- Numenius phaeopus (Linnaeus, 1758)

Known from the Carpathian Basin: Q4/I: Teufelslucken, Austria; Varbó-Lambrecht Kálmán Cave, Hungary; Detrekőszentmiklós-Pálffy Cave (Dzeráva Skála-Plavecky Mikulas), Slovakia (Lambrecht 1913, 1933, Soergel 1966, Jánossy 1979a, 1979b).

- Numenius sp.

Known from the Carpathian Basin: Q4/I: Hámor-Puskaporos Niche, Pilisszántói I. Niche (all in Hungary) (Lambrecht 1912, 1915, 1916, 1933, Jánossy 1979a, 1979b, 1986).

- Tringa totanus (Linnaeus, 1758)

Known from the Carpathian Basin: Q1-2: Betfia 5, Romania (Kessler 1975) Q4/I: Hámor-Herman Ottó Cave, Hungary; Ohábaponor-Bordu Mare Cave (Ohaba Ponor), Romania; Óruzsin-Antal Cave (Oruzer), Slovakia (Nehring 1880, Róth 1881, Lambrecht 1912, 1915, 1933, Kessler 1985, Jurcsák \& Kessler 1988, Gál 2002, 2003).

- Tringa glareola Linnaeus, 1758

Known from the Carpathian Basin: Q1: Betfia “Aven”, Romania (Kessler 1975, Jánossy 1979b, Gál 2002a) Q4/I: Gura Cheii Cave (Brașov), Romania (Jurcsák \& Kessler 1988).

- Tringa erythropus (Pallas, 1764)

Known from the Carpathian Basin: Q1: Betfia 9, Romania (Gál 2002a) Q4/I: Detrekőszentmiklós-Pálffy Cave (Dzeráva Skála-Plavecky Mikulas), Slovakia (Lambrecht 1913, 1933).

- Tringa nebularia (Gunnerus, 1767)

Known from the Carpathian Basin: Q1: Betfia 9, Romania (Gál 2002a).

- Tringa ochropus Linnaeus, 1758

Known from the Carpathian Basin: Q1: Betfia 9, Romania (Gál 2002a) Q3/I: Betfia 7/4, Romania (Gál 2002a, Jánossy 1979b, Kessler 1975).

- Tringa hypoleucos Linnaeus, 1758

Known from the Carpathian Basin: Q1: Teufelslucken, Austria; Betfia 2, 9, Romania (Kormos 1913, Čapek 1917, Lambrecht 1933, Kretzoi 1941, Soergel 1966, Kessler 1975, Jánossy 1979b, Gál 2002a) Q4/II: Körösbánlaki Cave, Romania (Kessler 1982).

- Tringa sp.

Known from the Carpathian Basin: Q1: Betfia 5, Romania (Kessler 1975) Q4/I: Budapest-Remetehegyi Niche, Hungary (Lambrecht 1933, Jánossy 1979a, 1979b) Q4/II: Grosse Offenbergerhöhle, Austria; Herkulesfürdő-Rablók Cave (Hoţilor Cave-Băile Herculane), Romania (Bocheński \& Tomek 1994, Gál 2002a).

- Scolopax rusticola Linnaeus, 1758

Known from the Carpathian Basin: Q3/I: Hundsheim, Austria (Jánossy 1971, 1974, 1979b) Q4/I: Velika Pecina; Vindija (all in Croatia); Budapest-Remetehegyi Niche, Hámor-Puskaporos Niche, Pilisszántói I. Niche (all in Hungary); Ohábaponor-Bordu Mare Cave (Ohaba Ponor), Romania; Detrekőszentmiklós-Pálffy Cave (Dzeráva Skála-Plavecky Mikulas), Slovakia (Lambrecht 1912, 1913, 1914, 1915, 1933, M. Malez 1961, V. Malez 1973, 1975, 1984, 1988, 1991, Jánossy 1979a, 1979b, 1986, Kessler 1985, Jurcsák \& Kessler 1988, Gál 2002a, 2003) Q4/II: Grosse Offenbergerhöhle, Teufelslucken (all in Austria); Csákvár-Esterházy Cave, Ecsegfalva 23, Felsőtárkány-Petényi Cave, Hillebrand Cave (Kőlyuk II), Legény Cave (all in Hungary); Kisbács-Bácsitorok (Baciu), Kőrösrévi Caves (Vadu Crișului 
Defile), Remetelórév-Bólyikői Cave (Piatra Boiului, Lorău), Vársonkolyos-Kis Magyar Cave (Şuncuiuș, Ungureasca Cave), Vársonkolyosi Caves (Şuncuiuṣ Defile) (all in Romania) (Lambrecht 1914, Kretzoi 1954, Soergel 1966, Kessler 1977, 1982, Jánossy 1979a, 1979b, Bocheński \& Tomek 1994, Kessler in Lazarovici \& Maxim 1995, Gál 2002a, 2007b, Pike-Tay et al. 2004).

- Scolopax sp.

Known from the Carpathian Basin: Q4/I: Teufelslucken, Austria; Novi I, III (Novi Caves), Slovakia (Nehring 1880, Róth 1881, Lambrecht 1912, 1933, Soergel 1966).

\section{Fam. Charadriidae Leach, 1820}

Plowers form the richest family of the order regarding to the number of species. They live near water, but they also have representatives preferring dry areas. Compared to the present number of species, we know of exceptionally few extinct ones. Only examples of extinct species from the Carpathian Basin were reported from the late Miocene of Hungary (Polgárdi) (Charadrius lambrechti Kessler, 2009 and Calidris janossyi Kessler, 2009) (Kessler, J. 2009b).

Recent species from the Carpathian Basin are known since the Quaternary.

- Arenaria interpres Linnaeus, 1758

Known from the Carpathian Basin: Q4/I: Petényi Cave, Hungary (Jánossy 1979a, 1979b).

- Philomachus pugnax Linnaeus, 1758

Known from the Carpathian Basin: Q1: Betfia 9, Romania (Gál 2002) Q4/I: Pilisszántói I. Niche, Tatabánya-Szelim Cave (all in Hungary) (Lambrecht 1915, 1933, Jánossy 1979a, 1979b, 1986).

- Charadrius hiaticula (Linnaeus, 1758)

Known from the Carpathian Basin: Q4/I: Teufelslucken, Austria (Soergel 1966).

- Charadrius sp.

Known from the Carpathian Basin: Q4/I: Szilvásvárad-Istállóskői Cave, Hungary (Jánossy 1952, 1955).

- Calidris alpina C. L. Brehm, 1822

Known from the Carpathian Basin: Q4/I: Pilisszántói I. Niche, Hungary; Detrekőszentmiklós-Pálffy Cave (Dzeráva Skála-Plavecky Mikulas), Slovakia (Lambrecht 1913, 1915, 1933, Jánossy 1979a, 1979b, 1986).

- Calidris ferruginea Merem, 1804

Known from the Carpathian Basin: Q4/I: Merkenstein, Austria (Wettstein \& Mühlhofer 1938).

- Vanellus vanellus (Linnaeus, 1758)

Known from the Carpathian Basin: Q1: Beremend 16, Hungary; Betfia 5, Romania (Lambrecht 1933, Kessler 1975, Jánossy 1992, 1996) Q4/I: Vindija, Croatia; Hámor-Puskaporos Niche, Pilisszántói I. Niche (all in Hungary) (Lambrecht 1912, 1915, 1933, M. Malez 1961, V. Malez 1973, 1988, 1991, Jánossy 1979a, 1979b, 1986) Q4/II: Teufelslucken, Austria; Legény Cave, Ordascsehi-Kistöltés (all in Hungary); Körösrévi Caves (Vadu Crișului Defile), Romania (Lambrecht 1914, Soergel 1966, Kessler 1982, Gál 2005, 2007a).

- Pluvialis squatarola (Linnaeus, 1758)

Known from the Carpathian Basin: Q4/I: Merkenstein, Austria; Esküllö-Igric Cave (Igreţ-Aștileu), Romania (Wettstein \& Mühlhofer 1938, Kessler 1985). 


\section{Fam. Recurvirostridae Bonaparte, 1854}

Avocets search for small invertebrates in shallow waters, with long legs and beaks. They nest on the coastal sand. Only one supposedly extinct species from Carpathian Basin is known from the early Pleistocene of Betfia, Transylvania (Romania) (Gál 2002).

Recent species from the Carpathian Basin are known since the Quaternary

- Recurvirostra avosetta Linnaeus, 1758

Known from the Carpathian Basin: Q4/I: Vindija, Croatia (M. Malez 1961, V. Malez 1973, 1988, 1991, V. Malez \& Rukavina 1979).

- Himantopus himantopus (Linnaeus, 1758)

Known from the Carpathian Basin: Q4/I: Pilisszántói I. Niche, Hungary (Lambrecht 1915, 1933, Jánossy 1979a, 1979b, 1986).

\section{Subord. Lari Sharpe, 1891}

\section{Fam. Lariidae Vigors, 1825}

Gulls and terns are spread out all across the globe, with a great number of species. They live on the shores of open waters. They feed on invertebrates, small vertebrates, carcasses, and some species near human settlements look for food on garbage heaps. They nest on coastal sand or walls of cliffs. Their nestlings are fluffy, but their parents feed them. Many species migrate, their young roam. Fossil species are not known from the Carpathian Basin.

Recent species from the Carpathian Basin are known since the Quaternary.

- Larus minutus Pallas, 1766

Known from the Carpathian Basin: Q3: Vindija, Croatia (M. Malez 1961, V. Malez 1973, 1988, 1991, V. Malez \& Rukavina 1979).

- Larus ridibundus Linnaeus, 1758

Known from the Carpathian Basin: Q1: Beremend 17, Hungary (Jánossy 1992, 1996) Q4/I: Merkenstein, Austria; Vindija, Croatia; Hámor Puskaporos Niche, Pilisszántói I. Niche, Répáshuta-Balla Cave (all in Hungary) (Lambrecht 1912, 1915, 1916, 1933, Wettstein \& Mühlhofer 1938, M. Malez 1961, V. Malez 1973, 1988, 1991, Jánossy 1979a, 1979b, 1986).

- Larus argentatus Pontoppidan, 1763

Known from the Carpathian Basin: Q4/II: Maroslele-Pana, Hungary (Bökönyi 1964, Jánossy 1979b, 1985, Gál 2004, 2007b).

- Larus canus Linneaus, 1758

Known from the Carpathian Basin: Q4/I: Detrekőszentmiklós-Pálffy Cave (Dzeráva Skála-Plavecky Mikulas), Slovakia (Lambrecht 1913, 1933).

- Larus sp.

Known from the Carpathian Basin: Q4/I: Vindija, Croatia (M. Malez 1961, V. Malez 1973, 1988, 1991, M. Malez \& Rukavina 1979).

- Sterna Hirundo Linnaeus, 1758

Known from the Carpathian Basin: Q4/I: Pilisszántói I. Niche, Hungary (Lambrecht 1915, 1933, Jánossy 1979a, 1979b, 1986).

- Sterna sp.

Known from the Carpathian Basin: Q1-2: Betfia 5, Romania (Kessler 1975). 
- Chlidonias niger (Linnaeus, 1758)

Known from the Carpathian Basin: Q1: Betfia 9, Romania (Gál 2002a).

- Chlidonias sp.

Known from the Carpathian Basin: Q4/I: Varbó-Lambrecht Kálmán Cave, Hungary (Jánossy 1979a).

\section{Conclusions}

The presented rich database grants the possibility to become familiar with the evolution and understanding of aquatic birds. They are well-presented, and even dominant, in avian fauna since the beginning of the Cretaceous, contrary to Sauriurae birds, where we do not know of species specifically living in wet environments neither from the Jurassic, nor from the Cretaceous. In the Paleogene, among recent birds (Ornithurae - Neornithes), they also constitute a majority compared to birds living in other environments, although this might also have taphonomic reasons, as shown by the avian fauna of Grube Messel.

In the case of most aquatic birds, the nestlings leave their nests early and start feeding autonomously. This is also a sign of maintaining old characteristics. Staying in their nests and being fed by their parents are either supposedly signs of typical nesting characteristics (for example with auks, penguins, where the fluffy nestlings cannot follow their parents to open waters due to various reasons), or the fact that the switch to living in aquatic environments is a secondary characteristic in their case. In contrary, a large number of the members of the listed group were or are not living in wet environments anymore (terrorbirds, Otididae, stone curlews etc.).

Another peculiarity while studying the database is that in the case of numerous groups (orders, families), the earliest representatives lived in entirely different - and rather distant environments than their current representatives. This is especially conspicuous in the case of flightless or barely flying types. This can have a number of possible reasons:

- the common ancestor could have formed before the dry "bridges" between the continents disappeared;

- becoming flightless is a subsequently formed characteristic;

- signs of being related were formed in an analogue way during evolution and their ancestral connection is only apparent.

The study of the database also makes it possible to mark the location where certain groups and types formed, and the direction in which they spread. The primer nature of Otididae in the Carpathian Basin is a typical example of this.

Data can, of course, be misleading, since finds of species that the fly well and roam large areas while immature can surface from rather unexpected places (as with the remains of seabirds).

The author is hoping to aid ornithologists and paleontologists in gaining knowledge on the geological history of the presence of taxons tied more or less to aquatic environments, trace the formation of certain units of taxonomy, and, especially, familiarize themselves with the ancient avian fauna of the Carpathian Basin. 


\section{Acknowledgements}

The author express his deep gratitude to Jenö Nagy, Lóránd Abos and József Vuts for the language revision.

\section{References}

Andrews, C. W. 1913. On some bird remains from the Upper Cretaceous of Transylvania. - Geological Magazine 10: 193-196.

Bindea, D. (ed.) 2008. Arheozoologia Transilvaniei in pre si protoistorie [Transylvanian archaeozoology in Praeand Protoistory]. - Trognost Cluj-Napoca (in Romanian and English)

Bocheński, Z. 1997. List of European fossil bird species. - Acta Zoologica Cracoviensia 40 (2): $293-333$.

Bocheński, Z. M. \& Tomek, T. 1994. Fossil and subfossil bird remains from five Austrian caves. - Acta Zoologica Cracoviensia 37: 347-358.

Bökönyi, S. 1964. A maroslele-panai neolithikus telep gerinces faunája [The Vertebrate fauna of the Neolithic site of Maroslele-Pana]. - Archeológiai Értesitő 91: 87-93 (in Hungarian)

Bökönyi, S. 1974. History of domestic mammals in Central and Eastern Europe. - Akadémiai Kiadó, Budapest

Bökönyi, S. 1984. Animal husbandry and hunting in Tác-Gorsium [The Vertebrate fauna of the Roman Town in Pannonia]. - Akadémiai Kiadó, Budapest (in Hungarian)

Bökönyi, S. \& Jánossy, D. 1965. Szubfosszilis vadmadár leletek Magyarországon (Subfossile Wildvogelfunde aus Ungarn) [Subfossil wild bird remains from Hungary]. - Vertebrata Hungarica 7: 85-99. (in Hungarian and German)

Brodkorb, P. 1963. Catalogue of fossil Birds. Part 1. (Archaeopterygiformes through Ardeiiformes). - Bulletin Florida State Museum, Biological Sciences 7: 179-293.

Brodkorb, P. 1964. Catalogue of fossil Birds. Part 2. (Anseriformes through Galliformes). - Bulletin Florida State Museum, Biological Sciences 8(3): 195-335.

Brodkorb, P. 1967. Catalogue of fossil Birds. Part 3. (Ralliformes, Ichthyornithiformes, Charadriiformes). - Bulletin Florida State Museum, Biological Sciences 2(3): 99-230.

Čapek, V. 1917. A püspökfürdői preglaciális madárfauna [The Preglaciale bird fauna from Püspökfürdő]. - Barlangkutatás 5: 66-74. (in Hungarian)

Dyke, G. J., Benton, M., Posmoseanu, E. \& Naish, I. 2010. Early Cretaceous (Berriasian) birds and pterosaurs from Cornet bauxite mine, Romania. - Paleontology 54(1): 79-95. DOI: 10.1111/j.1475-4983.2010.00997.x

Feduccia, A. 1999. The origin and evolution of birds. $2^{\text {nd }}$ edition. - New Haven, Yale University Press

Fischer, K. \& Stephan, B. 1977. Vogelknochenfunde aus Quartären Ablagerungen Südwestrumaniens [Bird bones from Quaternary deposits in Southwest Romania]. - Annalen für Ornithologie 1: 79-90. (in German)

Gál, E. 1998. Avifauna fosilă a peșterii Gura Cheii de la Râșnov (Jud Brașov) [Fossil bird fauna of the Gura Cheii Cave from Râșnov (Brașov County)]. - Studia Universitatis Babeș-Bolyai, Biologia 43(1-2): 88-93. (in Romanian)

Gál, E. 2001. Bird fauna of the Early Pleistocene site Betfia 9 from Romania. - In: $4^{\text {th }}$ Hungarian Palaeontological Session 4-5. Abstract volume: 14-15.

Gál, E. 2002a Avifauna pleistocena a Romaniei [Pleistocene bird faunas of Romania]. - (Unpublied C.Sc dissertation). - Universitatea din Bucureşti, Facultatea de Geologie Bucureşti (in Romanian)

Gál, E. 2002b Madárleletek a bajcsai várból [Bird finds from the Bajcsa Castle]. - In: Kovács, Gy. (ed.) Weitschawar Bajcsa-vár, pp. 101-105. Zalaegerszegi Múzeumok Igazgatósága, Zalaegerszeg (in Hungarian)

Gál, E. 2003. Bird remains from two Romanian caves: Curata Cave (Nandru) and Bordu Mare Cave (Ohaba Ponor). - ICAZ 2002. Abstracts, 56.

Gál, E. 2004. New evidence of fowling and poultry keeping in Pannonia, Dacia and Moesia during the period of the Roman Empire. - In: Gruppe, G. \& Peters, J. (eds.) Feathers, grit and symbolism. Birds and humans in the Ancient Old and New Worlds. - Proceedings of the $5^{\text {th }}$ Meeting of the ICAZ Bord Working Group, Munich, Germany, Documenta Archaeobiologiae 3. Verlag Marie Leidorf GmbH. Rahden/Westf., 301-316.

Gál, E. 2005. Animal remains from archaeological excavations in North-Eastern Hungary. - In: Gál, E., Juhász, I. \& Sümegi, P. (eds.) Environmental Archaeology in North-Eastern Hungary. - Varia Archaeologica Hungarica 19: 139-174. 
Gál, E. 2007a The Neolitic avifauna of Hungary within the context of the Carpathian Basin. - Antaeus 27: 273-286.

Gál, E. 2007b Fowling in lowlands. Neolitic and Chalcholitic bird exploitation in South-East-Romania and the Great Hungarian Plain. - Archaeolinqua, Series Minor 24. Budapest

Gál, E. 2008. Faunal and taphonomic approach to a Late Pleistocene cavern bird bone assemblage in North-West Hungary. - Géobios 41: 79-90.

Gál, E., Hír, J., Kessler, E., Kókay, J., Mészáros, L. \& Venczel, M. 1998-1999. Középső-miocén ősmaradványok, a Mátraszőlős, Rákóczi-kápolna alatti útbevágásból. I. A Mátraszőlős 1. lelőhely [Middle Miocene fossils from the sections at the Rákóczi Chapell at Mátraszőlős. I. Mátraszőlős 1. Site]. - Folia Historico Naturalia Musei Matraensis 23: 33-78. (in Hungarian with English Summary)

Gál, E., Hír, J., Kessler, E., Kókay, J. \& Venczel, M. 2000. Középső-miocén ősmaradványok a Mátraszőlős, Rákóczi-kápolna alatti útbevágásból. II. A Mátraszőlős 2. lelőhely [Middle Miocene fossils from the sections at the Rákóczi Chapell at Mátraszőlős. II. Mátraszőlős 2. site]. - Folia Historico Naturalia Musei Matraensis 24: 39-75. (in Hungarian with English Summary)

Gál, E \& Kessler, E. 2002. Bird remains from the Eneolithic and Iron Age site Bordușani-Popinã and Eneolithic site Hârșova (Southeast Romania). - Acta Zoologica Cracoviensia 45 (special issue): 253-262.

Göhlich, U. 2003. The avifauna of the Grund Beds (Middle Miocene, Early Badenian, Northern Austria). - Annalen des Naturhistorischen Museums in Wien 104A: 237-249.

Hamar, M. \& Csák, K. 1969. Contribuţii la cunoaşterea faunei de vertebrate pleistocene din Dealul Burzău (com. Rîpa, jud. Bihor) - jung Wurm [Contribution to the knowledge of Pleistocene vertebrate fauna from Burzau Hill (Village Rîpa, County Bihor) - Lower Würm]. - Studii şi cercetări biologice, Seria Zoologie 21(6): 425. (in Romanian)

Harrison, C. J. O. \& Walker, C. A. 1975. The Bradycnemidae, a new family of owls from the Upper Cretaceous of Romania. - Palaeontology 18(3): 563-570.

Hír, J., Kókay, J., Venczel, M., Gál, E. \& Kessler, E. 2001. Előzetes beszámoló a felsőtárkányi „Gödör-kert” őslénytani lelőhelykomplex újravizsgálatáról [A preliminary report on the revised investigation of the paleontological locality complex "Gödör-kert" at Felsőtárkány, Northern Hungary]. - Folia Historico-Naturalia Musei Matraensis 25: 41-65. (in Hungarian with English Summary)

Horáček, I. 1985. Survey of the fossil Vertebrate localities Včeláre 1-7. - Časopis pro Mineralogii a Geologii 30: 353-366.

Jánossy, D. 1952. Az Istállóskői barlang aurignaci faunája [The Aurignacian Cave fauna from Istállóskö]. - Földtani Közlöny 82(4-6): 181-203. (in Hungarian)

Jánossy, D. 1954. Fossile Ornis aus der Höhle von Istállóskő [Fossile birds from the Istállóskő Cave]. - Aquila 55-58: 205-223. (in German)

Jánossy, D. 1955. Die Vogel-und Saugetierreste der Spätpleistozänen Schichten der Höhle von Istállóskő [The bird and mammalian remains of the Late Pleistocene Layers of the Istállóskő Cave]. - Acta Archaeologica Academiae Sciencentiarum Hungaricae 5: 149-181. (in German)

Jánossy, D. 1960. Steinadler (Aquila chrysaetos L.) und Bartgeier (Gypaetus barbatus L.) aus dem Pleistozan Ungarns [Golden Eagle (Aquila chrysaetos L.) and Bearded Vulture (Gypaetus barbatus L.) from the Pleistocene of Hungary]. - Vertebrata Hungarica 2: 133-136. (in German)

Jánossy, D. 1962a Eine fossile Vogelfauna aus den Mosuterien-Schichten der Subalyuk-Höhle im Bükk-Gebirge [A fossil avifauna from the Mousterian layers of Subalyuk Cave in the Bükk Mountains]. - Aquila 67-68: 175-188. (in German)

Jánossy, D. 1962b Vorlaufige Mitteilung über die Mittelpleistozäne Vertebratenfauna der Tarkö-Felsnische (NO-Ungarn, Bükk-Gebirge) [Preliminary notification of the Middle Pleistocene vertebrate fauna of the Tarkő Rock (NE Hungary, Bükk Mountains)]. - Annales Historico Naturales Musei Nationalis Hungarici 54: 155-176. (in German)

Jánossy, D. 1962c Vorlaufige Ergebnisse der Ausgrabungen in der Felsnische Rejtek 1 (Bükk-Gebirge Gem. Répáshuta) [Preliminary reports of the excavation in Rejtek 1. hole (Bükk Mountains, Répáshuta village)]. Karszt- és Barlangkutatás 3: 49-58. (in German)

Jánossy, D. 1963. Die altpleistozäne Wirbeltierfauna von Kövesvárad bei Répáshuta (Bükk-Gebirge) [The Early Pleistocene vertebrate fauna of Kövesvárad at Répáshuta (Bükk Mountains)]. - Annales Historico Naturales Musei Nationalis Hungarici 55: 109-141. (in German)

Jánossy, D. 1964. Letzinterglaziale Vertebratenfauna aus der Kálmán Lambreht Höhle (Bükk-Gebirge, Nordost Ungarn) [Vertebrate fauna in the end of interglacial from Kálmán Lambrecht Cave (Bükk Mountains, Northeast Hungary)]. - Acta Zoologica Hungarica 9: 139-197. (in German) 
Jánossy, D. 1965. Fossiler Vogelfauna aus Mousterien Schichten der Curata-Hohle (Rumanien) [Fossil bird fauna from Mousterian layers of Curata Cave (Romania)]. - Vertebrata Hungarica 7(1-2): 106-116. (in German)

Jánossy, D. 1969. Új Eomyida (Rodentia, Mammalia) a bódvaszilasi Osztramosi kőfejtő 3. lelethelyének alsó-pleisztocén faunájából [New Eomyda (Rodentia, Mammalia) Lower Plesitocene fauna in Quarry site from Estramos 3. in Bódvaszilas]. - Öslénytani Viták 13: 5-40. (in Hungarian)

Jánossy, D. 1971. Die erste nachweis einer Kalt-Moustérien Vertebratenfauna in Ungarn (Tokod-Nagyberek, Komárom) [The first evidence of a Cold-Mousterian vertebrate fauna in Hungary (Tokod-Nagyberek, Komárom County)]. - Vertebrata Hungarica 12: 103-110. (in German)

Jánossy, D. 1972. Middle Pliocene Microvertebrate fauna from the Osztramos Loc.1 (Northern Hungary). - Annales Historico Naturales Musei Nationalis Hungarici 64: 27-48.

Jánossy, D. 1974. Die mittelpleistozäne Vogelfauna von Hundsheim (Niederösterreich) [The Middle Pleistocene avifauna of Hundsheim (Lower Austria)]. - Sitzungsberichte der Österreichischen Akademie der Wissenschaften, Mathematisch-Naturwissenschftliche Klasse, Abteilung I. 182: 211-257. (in German)

Jánossy, D. 1976a Plio-pleistocene bird remains from the Carpathian Basin. I. Galliformes 1. Tetraonidae. Aquila 82: 13-36.

Jánossy, D. 1976b Plio-pleistocene bird remains from the Carpathian Basin. II. Galliformes 2. Phasianidae. Aquila 83: 29-42.

Jánossy, D. 1976c Die Felsnische Tarkő und die Vertebratenfauna ihrer Ausfüllung [The cavity of Tarkő and the vertebrate fauna of their filling]. - Karszt- és Barlangkutatás 8: 3-106. (in German)

Jánossy, D. 1977. Plio-pleistocene bird remains from the Carpathian Basin. III. Strigiformes, Falconiformes, Caprimulgiformes, Apodiformes. - Aquila 84: 9-36.

Jánossy, D. 1978. Új finomrétegtani szint Magyarország pleisztocén őslénytani sorozatában [A new fine stratigrafic level in Paleontological series at Hungarian Pleistocene]. - Földrajzi Közlemények 26(1-3): 161-174. (in Hungarian)

Jánossy, D. 1979a A magyarországi pleisztocén tagolása a gerinces faunák alapján [Layout of the Hungarian Pleistocene based on the Vertebrate fauna]. - Akadémiai Kiadó, Budapest (in Hungarian)

Jánossy, D. 1979b Plio-pleistocene bird remains from the Carpathian Basin. IV. Anseriformes, Gruiformes, Charadriiformes, Passeriformes. - Aquila 85: 11-39.

Jánossy, D. 1979c Plio-pleistocene bird remains from the Carpathian Basin. V. Podicipediformes, Ciconiiformes, Otidiformes, Columbiformes, Piciformes. - Aquila 86: 19-33.

Jánossy, D. 1980. Plio-pleistocene bird remains from the Carpathian Basin. VI. Systematical and Geographical Catalogue. - Aquila 87: 9-22.

Jánossy, D. 1981. Die altpleistozänen Vogelfaunen von Deutsch-Altenburg 2 und 4 (Niederösterreich) [The Early Pleistocene bird fauna of Deutsch-Altenburg 2 and 4 (Lower Austria)]. - Beiträge zur Palaontologie von Österreich 8: 375-391. (in German)

Jánossy, D. 1982. The extinct ancestor of Anser anser in Europe. - Aquila 88: 21-22.

Jánossy, D. 1983. Lemming-remain from the Older Pleistocene of Southern Hungary (Villany, Somssich-hegy 2.). - Fragmenta Mineralogica et Paleontologica 11: 55-60.

Jánossy, D. 1985. Wildvogelreste aus archaeologischen Grabungen in Ungarn (Neoliticum bis Mittelalter) [Wild bird remains from archaeological excavations in Hungary (from the Neoliticum to the Middle Ages)]. - Fragmenta Mineralogica et Paleontologica 12: 67-103. (in German)

Jánossy, D. 1986. Pleistocene Vertebrate faunas of Hungary. - Akadémiai Kiadó, Budapest \& Elsevier, Amsterdam

Jánossy, D. 1987b Ältestpleistozäne vertebratenfauna von Beremend 15 (Sud-Ungarn) [Earliest Pleistocene vertebrate fauna of Beremend $15^{\text {th }}$ (South Hungary)]. - Fragmenta Mineralogica et Paleontologica 13: 89-96. (in German)

Jánossy, D. 1990. Vertebrate fauna of site II. - In: Kretzoi, M. (ed.) Vértesszőlős: site, man and culture. - Akadémiai Kiadó, Budapest, pp. 187-229.

Jánossy, D. 1991. Late Miocene bird remains from Polgárdi (W-Hungary). - Aquila 98: 13-35.

Jánossy, D. 1992. Lower Pleistocene bird remains from Beremend (S-Hungary, Loc. 15. and 16.). - Aquila 99: 9-25. Jánossy, D. 1993. Bird remains from the Upper Miocene (MN9) of Rudabánya (N-Hungary). - Aquila 100: 53-70.

Jánossy, D. 1995. A Late Miocene avifauna from Polgárdi, Western Hungary. - Acta Palaeornithologica, Courier Forschungsinstitut Senckenberg 181: 203-206.

Jánossy, D. 1996. Lower Pleistocene vertebrate faunas from the localities 16. and 17. of Beremend (Southern Hungary). - Fragmenta Mineralogica et Paleontologica 18: 91-102. 
Jánossy, D. \& Kordos, L. 1976a Az Osztramos gerinces lelőhelyeinek faunisztikai és karszt-morfológiai áttekintése (1974-ig) [The faunistical and karst-morphological review of paleontological localities for Vertebrates at Osztramos (to 1974)]. - Fragmenta Mineralogica et Paleontologica 8: 39- 92. (in Hungarian and English)

Jánossy, D. \& Kordos, L. 1976b Pleistocene-Holocene Mollusc and Vertebrate fauna of two caves in Hungary. Annales Historico Naturales Musei Nationalis Hungarici 68: 5-29.

Jánossy, D. \& Kordos, L. 1977. Az Osztramos gerinces lelőhelyeinek faunisztikai és karsztmorfológiai áttekintése (1975-ig) [The faunistical and karst-morphological review of paleontological localities for Vertebrates at Osztramos (to 1975)]. - Fragmenta Mineralogica et Palaeontologica 8: 39-72. (in Hungarian and English)

Jánossy, D. \& Vörös, I. 1987. Die mittelpleistozäne Fauna der Höhle des Hungaria-Berges bei Dorog (Gerecse Gebirge, Ungarn) [The Middle Pleistocene fauna of the cave of the Hungarian Mountain at Dorog (Gerecse Mountain, Hungary)]. - Fragmenta Mineralogica et Paleontologica 13: 97-110. (in German)

Jarvis, E. D., Mirarab, S., Aberer, A. J., Li, B. et al. 2014. Whole-genome analyses resolve early branches in the tree of life of modern birds. - Science 346(6215): 1320-1331. DOI: 10.1126/science.1253451

Jurcsák, T. 1974. Prezenţa lui Gyps fulvus (Habl.) în cuaternarul României [The presence of Gyps fulvus (Habl) in Romanian Queternar]. - Nymphaea 2: 169-184. (in Romanian)

Jurcsák, T. \& Kessler, E. 1973. Cercetări paleornitologice din România [Paleornithological researces in Romania]. - Nymphaea 1: 169-179. (in Romanian)

Jurcsák, T. \& Kessler, E. 1986. Evoluţia avifaunei pe teritoriul României (I) [Evolution of the avifauna in the territory of Romania. Part I.]. - Crisia 16: 577-615. (in Romanian with English Summary)

Jurcsák, T. \& Kessler, E. 1988. Evoluţia avifaunei pe teritoriul României (III) [Evolution of the avifauna]. - Crisia 18: 647-688. (in Romanian with English Summary)

Kessler, E. 1974a Date noi asupra avifaunei fosile a Dealului Burzău - Râpa [New data to fossil bird fauna from Burzau Hill (Village Râpa)]. - Nymphaea 2: 159-167. (in Romanian)

Kessler, E. 1974b Contribuţii noi la avifauna depozitelor hidrotermale postglaciare de la Băile 1 Mai - Oradea, jud. Bihor [New contributions to postglaciale avifauna and hydrothermal sediments from Băile 1 Mai Oradea, Bihor County]. - Tibiscus 11: 123-128. (in Romanian)

Kessler, E. 1974c Avifauna postglaciară de la Cuina Turcului, Cazanele Mari, România [Postglacial avifauna from Cuina Turcului, Cazanele Mari, România]. - Tibiscus 11: 113-122. (in Romanian)

Kessler, E. 1975. Contribuţii noi la studiul avifaunei fosile de la Betfia, jud. Bihor [New contributions to fossile avifauna from Betfia, Bihor County]. - Nymphaea 3: 53-59. (in Romanian)

Kessler, E. 1977a Noi date privind avifauna pleistocenului superior din România [New data to Late Pleistocene fossil bird fauna from Romania]. - Nymphaea 5: 95-99. (in Romanian)

Kessler, E. 1977b Contribuții la studiul prezenţei genului Pyrrhocorax în avifauna fosilă a României [Contributions to the knowledge of the presence of Pyrrhocorax genus in fossil bird fauna in Romania]. - Nymphaea 5: 100-105. (in Romanian)

Kessler, E. 1977c Avifauna postglaciară de la Vadu Crişului, jud. Bihor [Postglaciale avifauna from Vadu Crişului, Bihor County]. - Tibiscus 14: 255-260. (in Romanian)

Kessler, E. 1977d Avifauna zonei Crişurilor din perioada pliocenului până astăzi [Avifauna of Cris area from Pliocene to today]. - Unpublished C.Sc dissertation, Universitatea din Bucureşti, Facultatea de Biologie (in Romanian)

Kessler, E. 1978a Îmbogăţirea inventarului paleoavifaunistic al României între anii 1973-1978 [Enrichment of the paleornithological inventory of Romania between 1973-1978]. - Nymphaea 6: 165-180. (in Romanian)

Kessler, E. 1978b Contribuţii noi privind prezenţa genului Pelecanus în avifauna sub-fosilă a României [New contributions about Pelecanus species presence in the subfossil avifauna of Romania]. - Nymphaea 6: 181182. (in Romanian)

Kessler, E. 1980-1981. Noi date privind avifauna pleistocenă a Văii Ierului (jud. Bihor) [New data on the Pleistocene bird fauna of Valea Ierului (Bihor County)]. - Nymphaea 8-9: 259-264. (in Romanian)

Kessler, E. 1982. Avifauna fosilă şi subfosilă a Munţilor Apuseni [Fossil and subfossil bird fauna from Apuseni Mountains]. - Nymphaea 10: 171-181. (in Romanian)

Kessler, E. 1984. Noi contribuţii privind studiul avifaunelor din Paratethys [New contributions to the study of bird faunas from Paratethys]. - Crisia 14: 521-532.

Kessler, E. 1985a Material fosil şi subfosil de păsări din Defileul Dunării de la Porţile de Fier [Fossil and subfossil bird remains of Iron Gates from Danube Gorge]. - Drobeta 1985: 337-339. (in Romanian)

Kessler, E. 1985b Contribuţii noi la studiul avifaunelor cuaternare din România [New contributions to the study of Quaternary bird faunas in Romania]. - Crişia 15: 485-491. (in Romanian) 
Kessler, E. 1995. Păsări [Birds]. - In: Lazarovici, Gh. \& Maxim, Z. (eds.) 1995. Gura Baciului. Monografie arheologica [Gura Baciului. Archaeological monograph]. - Bibliotheca Musei Napocensis. XI. Cluj-Napoca (in Romanian)

Kessler, E. 2009. The oldest modern bird (Ornithurinae) remains from the Early Oligocene of Hungary. - Fragmenta Palaeontologica Hungarica 27: 93-96.

Kessler, E. 2013a Neogene songbirds (Aves, Passeriformes) faunae from Hungary. - Hantkeniana 2013: 37-149.

Kessler, E. 2013b Early bird evolution in context with Cornet's (Berriasian Lower Cretaceous, Romania) taxa (2013 manuscript)

Kessler, E. \& Codrea, V. 1996. A bird form the Middle Miocene (Badenian) of Zarand depression (Western Romania). - Studia University Babeş-Bolyai, Series Geologia 41(2): 127-130.

Kessler, E., Codrea, V. \& Vremir, M. 1998. A fossil bird from the Lower Oligocene of Cluj-Napoca (Western Romania). - Studia University Babes-Bolyai, Series Geologia 43(2): 7-12.

Kessler, E. \& Gál, E. 1997. Resturi fosile şi subfosile de păsări din Banat [Fossil and subfossil bird remains in Banat]. - Analele Banatului - Ştiinţele Naturii 3: 141-144. (in Romanian with English Summary)

Kessler, E. \& Gál, E. 1998. Resturi fosile şi subfosile de păsări în siturile paleolitice şi neolitice din Cheile Turzii şi Cheile Turenilor (Județul Cluj) [Fossil and subfossil bird remains in Paleolitical and Neolitical sites from Cheile Turzii and Cheile Turenilor (Cluj County)]. - Angvistia 3: 9-122. (in Romanian with English Summary)

Kessler, E., Grigorescu, D. \& Csiki, Z. 2005. Elopteryx revisited - a new bird-like specimen from the Maastrichtian of the Hateg Basin (Romania). - Acta Paleontologica Romaniae 5: 249-258.

Kessler, E. \& Hír, J. 2009. A new Anserid species from the Neogene of Hungary. - Fragmenta Palaeontologica Hungarica 27: 97-101.

Kessler, E. \& Jurcsák, T. 1984. Fossil bird remains in the bauxite from Cornet (Romania, Bihor County). Travaux du Muséum d'Histoire naturelle 'Grigore Antipa' București 25: 393-401.

Kessler, E. \& Jurcsák, T. 1986. New contributions to the knowledge of the Lower Cretaceous bird remains from Cornet (Romania). - Travaux du Muséum d'Histoire naturelle 'Grigore Antipa' București 27: 289-295.

Kessler, J. 2009a Új eredmények a Kárpát-medence neogén és negyedidőszaki madár-világához, I. rész [New results with regard to the Neogene and Quaternary avifauna of the Carpathian Basin, Part I.]. - Földtani Közlöny 139(1): 67-82. (in Hungarian with English Summary)

Kessler, J. 2009b Új eredmények a Kárpát-medence neogén és negyedidőszaki madár-világához, II. rész [New results with regard to the Neogene and Quaternary avifauna of the Carpathian Basin, Part II.]. - Földtani Közlöny 139(3): 251-271. (in Hungarian with English Summary)

Kessler, J. 2010. Új eredmények a Kárpát-medence neogén és negyedidőszaki madár-világához, III. rész [New results with regard to the neogene and Quaternary avifauna of the Carpathian Basin, Part III.]. - Földtani Közlöny 140(1): 53-72. (in Hungarian with English Summary)

Kessler, J. 2013. A Kárpát-medence madárvilágának őslénytani kézikönyve [Paleontological handbook of birdlife in the Carpathian Basin]. - Könyvmühely, Miskolc (in Hungarian)

Kessler, J. \& Hír, J. 2012a Észak-Magyarország madárvilága a miocénben I. [The avifauna in North Hungary during the Miocene. Part I.]. - Földtani Közlöny 142(1): 67-78. (in Hungarian with English Summary)

Kessler, J. \& Hír, J. 2012b Észak-Magyarország madárvilága a miocénben II. [The avifauna in North Hungary during the Miocene. Part II.)]. - Földtani Közlöny 141(2): 149-168. (in Hungarian with English Summary)

Kessler, J. \& Rabi, M. (manuscript) Máriahalom felső-oligocén madárfaunája [Upper Oligocene bird fauna from Máriahalom, Pest County, Hungary]. (in Hungarian)

Kordos, L. 1984. A bodajki Rigó-lyuk újholocén kitöltésének vizsgálata [Examination of Bodajk Rigó-hole filling in Holocene]. - Folia Musei Historico-Naturalis Bakonyensis 3: 31-42. (in Hungarian)

Kormos, T. 1913. Kleinere Mitteilungen aus dem ungarischen Pleistozän [Small releases from the Hungarian Pleistocene]. - Centralblatt für Mineralogie, Geologie und Palaontologie 1913: 13-17. (in German)

Kormos, T. 1914a Az 1913. évben végzett ásatásaim eredményei [The results of the excavations carried in the year 1913]. - A Magyar Királyi Földtani Intézet 1913. évi jelentése, pp. 498-505, 531-540. (in Hungarian)

Kormos, T. 1914b Új adatok a hidegszamosi csontbarlang faunájához [New data of 'Bone Cave' fauna from Hidegszamos]. - Barlangkutatás 2(3): 136-137. (in Hungarian)

Kormos, T. 1915. A Devence-barlangi praehistoricus telep Biharvármegyében [The prehistorical site in Devence Cave at Bihar County]. - Barlangkutatás 3-4: 153-163. (in Hungarian)

Kormos, T. 1916. Újabb ásatások az Igric-barlangban [New excavations in Igric Cave]. - Magyar Királyi Földtani Intézet 1915. évi jelentése, pp. 558-567. (in Hungarian) 
Kormos, T. 1917. Új adatok a püspökfürdői Somlyó-hegy preglaciális faunájához [New data to preglaciale fauna of Somlyó Hill from Püspökfürdő]. - Állattani Közlöny 27(1-2): 40-62. (in Hungarian)

Körösi, A. (Manuscript) Lussonium - Paks-Dunakömlőd római erőd állatcsont maradványai. Szarvasmarhák és kiskérődzők [Lussonium Paks-Dunakömlőd animal bone remains of a Roman fort. Cattle and small ruminants]. - Pécs, János Pannonius Egyetem (in Hungarian)

Kretzoi, M. 1941. Ösemlős maradványok Betfiáról [Remains of fossil Mammals from Betfia]. - Földtani Közlöny 71(7-12): 239-245 (in Hungarian)

Kretzoi, M. 1954. Befejező jelentés a csákvári barlang őslénytani feltárásáról [Final report of paleontological exploratoion of the Csákvár Cave]. - A Magyar Állami Földtani Intézet Évi Jelentése az 1952 évről, pp. 3769. (in Hungarian)

Kretzoi, M. 1956. A Villányi hegység alsó-pleisztocén gerincesfaunái [Ostrich remains from Kisláng, Fejér County]. - Geologica Hungarica 27: 1-264. (in Hungarian)

Kretzoi, M. 1957. Madármaradványok a Csákvári faunából [Fossil bird remains from Csákvár Fauna]. - Aquila 63-64: 239-248. (in Hungarian)

Kretzoi, M. 1961-62. Madár-maradványok a betfiai alsópleisztocén faunából [Bird remains in the Lower Pleistocene fauna from Betfia]. - Aquila 67-68: 167-174. (in Hungarian)

Krolopp, E. \& Vörös, I. 1982. Macro-Mammal und Mollusca Reste des Torflagers Mezőlak - Szélmező puszta [Macro-Mammal and Mollusc remains of Mezőlak - Szélmező Puszta]. - Folia Musei Historico-naturalis Bakonyiensis 1: 39-64. (in German)

Kuročkin, E. N. 1995. Synopsis of Mesozoic birds and early evolution of Class Aves. - Archaeopteryx 13: 47-66.

Kuročkin, E. N. 2006. Parallel evolution of theropod dinosaurs and birds. - Entomological Review 86 (Suppl.1): $45-58$.

Lambrecht, K. 1912a A borsodi Bükk fossilis madarai - Fossile Vögel des Borsoder Bükk-Gebirges [Fossil birds of the Bükk Mountains from Borsod]. - Aquila 19: 270-287. (in Hungarian and German)

Lambrecht, K. 1912b Magyarország fossilis madarai - Die fossilen Vögel Ungarns [The Fossil birds of Hungary]. - Aquila 19: 288-320. (in Hungarian and German)

Lambrecht, K. 1913. Neueren Daten zur fossilen Ornis unseren Höhlen [Recent data for fossil birds in our caves]. - Barlangkutatás 1-173. (in German)

Lambrecht, K. 1915. Fossilis nagy fülesbagoly (Bubo maximus Flemm.) és egyéb madármaradványok a magyarországi pleistocénből [Fossil's Big Eared Owl (Bubo maximus Flemm.) and other bird remains in Hungarian Pleistocene]. - Aquila 22: 177-187. (in Hungarian)

Lambrecht, K. 1916a Az első magyar preglaciális madárfauna - Die erste ungarische praglaziale Vogelfauna [The first Hungarian Preglaciale bird fauna]. - Aquila 22: 165-172. (in Hungarian and German)

Lambrecht, K. 1916b Die gattung Plotus im ungarischen Neogen [The Plotus genus in the Hungarian Neogene]. - Mitteilungen aus dem Jahrbuche der Königlich Ungarischen Geologischen Reichsanstalt 24: 1-4. (in German)

Lambrecht, K. 1929. Mezozoische und tertiäre Vogelreste aus Siebenburgen [Mezozoic and Tertiary bird remains from Transylvania]. - In: Csiki, E. (ed.) X. Congres International de Zoologie, pp. 1262-1275. (in German)

Lambrecht, K. 1931. Cygnopterus und Cygnavus zwei fossile Schwane aus dem Tertiar Europas [Cygnopterus and Cygnavus, two ossil swans from the Tertiary of Europe]. - Bulletin du Musée Royal d'Histoire naturalle de Belgique 7(31): 1-6. (in German)

Lambrecht, K. 1933. Handbuch der Palaeornithologie [Handbook of Palaeornithology]. - Gebrüder Borntraeger, Berlin (in German)

Lóczy, L. 1877. A Baráthegyi barlang megvizsgálásáról [The examination of the Baráthegyi Cave]. - Természettudományi Közlöny 89: 15. (in Hungarian)

Malez, M. 1961. Pecinska hijena iz indije kod Voce [The Cave Hyaenas come from Vindija Cave]. - Geologiceskij Vjesnik 14: 221-244. (in Croatian)

Malez, M. \& Rukavina, D. 1975. Kristurbacijske pojave u gornjopleistocenskim nasla-gama pecine Vindije kod Donje Voce u sjeverozapadnoj Hrvatskoj [Cristurbation appearance in Upper Pleistocene at Vindija Cave in Northwest Croatia]. - Rad Hazu (Jugoslavenske Akademije Znamosti Umjamosti) 371: 245-265 (in Croatian)

Malez, V. 1973. Fosilne ptice Jugoslavije [Fossil birds from Yugoslavia]. - Unpublished Dissertation, Zagreb University (in Croatian)

Malez, V. 1984. Paleornitološki ostaci iz kvartarnih naslaga nekih spilja Hrvatske i Slovenije [Paleornithological remains from the Quaternary Sediments of some Croatian and Slovenian caves]. - In: Deveti jugoslavenski speleoloski kongres, Zbornik predavanja 711-719. (in Croatian) 
Malez, V. 1986. Paleoornitološka istrazivanja u našim krajevima [Paleornithological research in our region]. - In: Mestrov, M. \& Sušić, G. (eds.) Ornitologija u Hrvatskoj, pp. 205-212. (in Croatian)

Malez, V. 1988. Pleistocenska ornitofauna iz spilje Vindije u sjevernozapadnoj Hrvatskoj [Pleistocene bird fauna from Vindija Cave in Northwestern Croatia]. - Rad Hazu (Jugoslavenske Akademije Znanosti i Umjetnosti), Varazdin 2: 31-203. (in Croatian)

Malez, V. 1993. Otis tarda L. i Otis tetrax L. u kvartarnim naslagama Hrvatske [Otis tarda L. and Otis tetrax L. in Croatian Quaternary sediments]. - Rad Hazu (Jugoslavenske Akademije Znamosti Umjamosti) 463: 63-79. (in Croatian)

Malez-Bačić, V. 1979. Pleistocenska ornitofauna iz Šandalje u Istri te njezino stratigrafsko i paleoekološko značenje [Pleistocene bird fauna from Šandalja in Istria and its stratigraphic and paleoecological significance]. - Palaeontologia Jugoslavica 21: 1-46. (in Croatian)

Mayr, G. 2009. Paleogene Fossil Birds. - Springer Verlag Berlin, Heidelberg, DOI: 10.107/978-3-540-89628-9

Milne-Edwards, A. 1867-68. Recherches anatomiques et paléontologiques pour servir àl'histoire des oiseaux fossiles de la France, Vol. 1. [Anatomical and paleontological research to serve the history of fossil birds of France, Vol. 1.]. - Victor Masson et Fils, Paris (in French)

Milne-Edwards, A. 1869-71. Recherches anatomiques et paléontologiques pour servir àl'histoire des oiseaux fossiles de la France, Vol. 2. [Anatomical and paleontological research to serve the history of fossil birds of France, Vol. 2.]. - Paris, G. Masson (in French)

Mlikovskỳ, J. 1992. Late Miocene birds of Götzendorf/Leitha, Austria. - Annalen des Naturhistorischen Museums in Wien (A) 91: 97-100.

Mlikovskỳ, J. (ed.) 1996. Tertiary avian localities of Europe. - Acta Universitatis Carolinae, Geologica 39: 517848.

Mlikovskỳ, J. 1997 A new tropical bird (Aves: Phaethontidae) from the Late Miocene of Vösendorf, Austria. - Annalen des Naturhistorischen Museums in Wien (A) 98: 151-154.

Mlikovskỳ, J. 1998 Early Pleistocene birds of Deutsch-Altenburg, Austria. - Acta Societatis Zoologicae Bohemicae 62: 135-141.

Mlikovskỳ, J. 2002. Cenozoic birds of the World. Part 1: Europe. - Ninox Press, Praha

Mlikovskỳ, J. 2007. Taxonomic identity of Eostega Lebedinskyi Lambrecht, 1929 (Aves) from the Middle Eocene of Romania. - Annalen des Naturhistorischen Museums in Wien 109A: 19-27.

Mlikovskỳ, J. 2009. Middle Pleistocene birds of Hundsheim, Austria. - Journal of the National Museum (Prague), Natural History Series 177(7): 69-82.

Mourer-Chauviré, C. 1995. Dynamics of the avifauna during the Paleogene and the Early Neogene of France. Settling of the recent fauna. - Acta Zoologica Cracoviensia 38(3): 325-342.

Mottl, M. 1938. Faunen, Flora und Kultur des ungarische Solutréen [Fauna, flora and culture of the Hungarian Solutréen]. - Quartär 1: 36-54. (in German)

Mottl, M. 1941. Die Interglazial- und Interglazialzeiten im Lichte der Ungarischen Saugetierfauna [Interglacial and interglacial times in the light of Hungarian mammalian fauna]. - Mittlheilunge Jahrbuche Königlich Ungarische Geologisches Institut 35: 13-39. (in German)

Nehring, A. 1880. Uebersicht über vierundzwanzig mitteleuropäische Quartär-Faunen [Survey of twenty-four Central European Quaternary fauna]. - Zeitschrift der Deutschen Geologischen Gesellschaft 32: 468-509. (in German)

Nehring, A. 1881. Dr. Roth's Ausgrabungen in Oberungarischen Höhlen [Dr. Roth's excavations in North Hungarian caves]. - Zeitschrift für Ethnologie 13: 96-109. (in German)

Olson, S. L. 1977. A synopsis of the fossil Rallidae. - In: Ripley, S. D. (ed.) Rails of the world, Boston, pp. 339373.

Olson, S. L. 1985. The fossil record of birds. - Avian Biology 7: 80-252.

Róth, S. 1881. Szepesmegye néhány barlangjának leirása [Description of some caves in Szepes County]. - Mathematikai és Természettudományi Közlemények 16: 613-648. (in Hungarian)

Soergel, E. 1966. Die Vogelreste [The Bird Remains]. - In: Ehrenberg, K. (ed.) Die Teufels-oder Fuck-senlucke bei Eggenburg (NÖ) [The Devil or Fuck senlucke at Eggenburg (NA)]. - Denkschriften der Mathematisch-Naturwissenschftliche Klasse der Österreichischen Akademie der Wissenschaften, pp. 93-107. (in German)

Sibley, Ch. G. \& Ahlquist, J. E. 1990. Phylogeny and classification of birds. - Yale University Press, New Haven, Conn

Sibley, Ch. G. \& Monroe, B. L. Jr. 1990. Distribution and taxonomy of birds of the world. - Yale University Press, New Haven, Conn 
Téglás, G. 1880. A nándori barlangcsoport Hunyad megyében [The Nándori Cave-group in Hunyad County]. Természettudományi Közlöny 12: 303-315. (in Hungarian)

Toula, F. 1909. Diluviale Säugetierreste vom Gesprengberg, Kronstadt in Siebenbürgen [Diluvial Mammal remains from Gesprengberg Cave, Brassó in Transylvania]. - Jahrbuch der Königlich Geologische Reichsanstalt 59(3-4): 575-614. (in German)

Tyrberg, T. 1998. Pleistocene birds of the Palearctic: a catalogue. - Cambridge, Mass.: Nuttall Ornithological Club [Publications of the Nuttall Ornithological Club, No. 27.]

Wetmore, A. 1960. A classification for the birds of the world. - Smithsonian Miscellaneous Collections 139 (11): $1-37$.

Wettstein, O. V. \& Mühlhofer, F. 1938. Die Fauna der Höhle von Merkenstein in NÖ [The fauna of the Merkenstein Cave in North Austria]. - Archiv Naturgeschichte, new series 7(4): 514-558. (in German)

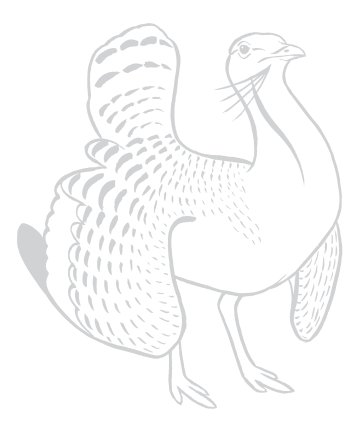

